\title{
Remote Asymmetric Induction in an Intramolecular Ionic Diels-Alder Reaction: Application to the Total Synthesis of (+)-Dihydrocompactin
}

\author{
Tarek Sammakia,* Deidre M. Johns, Ganghyeok Kim, and Martin A. Berliner \\ Department of Chemistry and Biochemistry \\ University of Colorado, Boulder, Colorado 80309-0215 \\ Email: sammakia@colorado.edu
}

\section{Supporting Information}

Table of Contents

S1 Synthesis of intramolecular Diels-Alder substrate $\mathbf{5}$

S3 Materials and methods, Experimental procedure for the synthesis of S-15,

S4 Experimental procedures for the synthesis of S-17and S-18

S5 Experimental procedures for the synthesis of $\mathbf{S - 1 9}$ and $\mathbf{S}-\mathbf{2 1}$

S6 Experimental procedures for the synthesis of $\mathbf{S - 2 2}$ and $\mathbf{S - 2 3}$

S7 Experimental procedures for the synthesis of $\mathbf{S - 2 5}$ and $\mathbf{S - 2 6}$

S8 $\quad$ Experimental procedures for the synthesis of S-27 and $\mathbf{S - 2 8}$

S9 Experimental procedures for the synthesis of $\mathbf{S - 2 9}$ and $\mathbf{S - 3 0}$

S10 Experimental procedures for the synthesis of $\mathbf{5}$ and $\mathbf{1 1}$

S11 Experimental procedure for the synthesis of S-31

S12 Experimental procedures for the synthesis of S-32 and $\mathbf{1 5}$

S13 Experimental procedures for the synthesis of S-33 and $\mathbf{S - 3 4}$

S14 Experimental procedures for the synthesis of $\mathbf{1 6}$ and dihydrocompactin

S15 Comments on the molecular modeling of the oxocarbenium ion intermediate

S16 X-ray crystallography data for compound ent-11 (separate supporting info file)

S26 $\quad{ }^{1} \mathrm{H}$ and ${ }^{13} \mathrm{C}$ NMR spectra for selected compounds and gDQCOSY for dihydrocompactin (separate supporting info file)

\section{Synthesis of Intramolecular Diels-Alder Substrate 5:}

Our synthesis of the key intramolecular Diels-Alder substrate (5) began with the hydroalumination of 5-chloro-1-pentyne with DIBAL-H and iodination of the intermediate vinyl alane (S-14) with iodine to provide vinyl iodide $\mathbf{S - 1 5}{ }^{1}$ (Scheme S-1). This compound was subjected to a Negishi coupling with the vinyl alane S-16, derived from the hydroalumination of propyne, to provide chlorodiene $\mathbf{S - 1 7 ,}{ }^{2}$ which upon treatment with $\mathrm{NaI}$ in acetone provided iododiene S-18. ${ }^{3}$ One carbon homologation of $\mathbf{S - 1 8}$ was accomplished by lithiation of the iodide with $s e c$-BuLi and trapping with DMF to provide dienal S-19. ${ }^{4}$

Scheme S-1
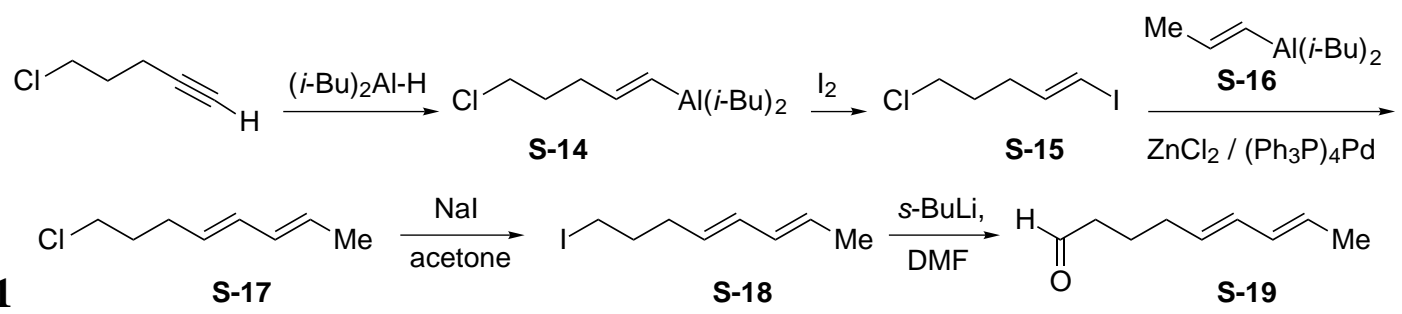

(1) Brown, H. C.; Subrahmanyam, C.; Hamaoka, T.; Ravindran, N.; Bowman, D. H.; Misumi, S.; Unni, M. K.; Somayaji, V.; Bhat, N. G. J. Org. Chem. 1989, 54, 6068.

(2) Karanewsky, D. S.; Badia, M. C. Tetrahedron Lett. 1993, 34, 39.

(3) Grieco, P. A.; Kaufman, M. D. J. Org. Chem. 1999, 64, 6041.

(4) Craig, D.; Fischer, D. A.; Kemal, O.; Marsh, A.; Plessner, T.; Slawin, A. M. Z.; Williams, D. J. Tetrahedron, 1991, 47, 3095. 
The coupling partner for dienal S-19, alkyne S-26, was prepared as described in Scheme S2. Asymmetric crotylation of $\beta$-benzyloxypropanal (S-20) by the method of Nokami ${ }^{5}$ provided compound S-21 which was subjected to ozonolytic cleavage of the alkene followed by a reductive workup $\left(\mathrm{NaBH}_{4}\right)$ to provide diol $\mathbf{S - 2 2} .6$ This compound was then protected as the acetonide to provide $\mathbf{S - 2 3}$, and the benzyl protecting group removed by hydrogenolysis with Pearlman's catalyst to provide $\mathbf{S - 2 4} .^{7}$ The primary alcohol of $\mathbf{S - 2 4}$ was activated as the tosylate to provide $\mathbf{S - 2 5}$, and then displaced with sodium acetylide in DMSO to provide $\mathbf{S - 2 6 .}{ }^{8}$

\section{Scheme S-2}
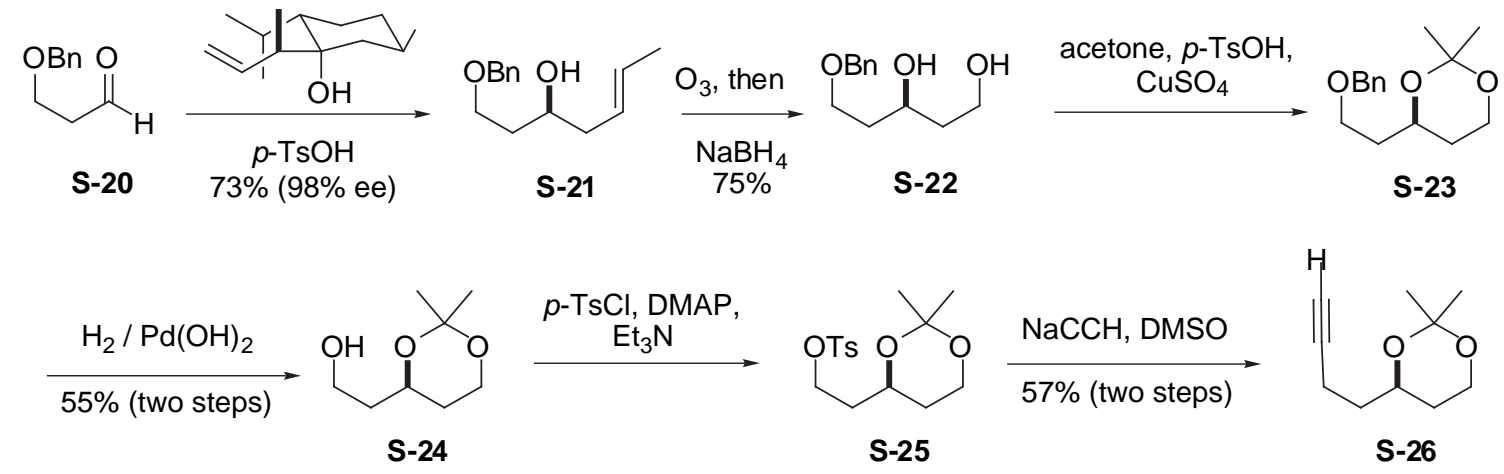

Coupling of S-19 and S-26 was accomplished as described in Scheme S-3. Metallation of S-26 with $n$-BuLi and addition to aldehyde $\mathbf{S - 1 9}$ provided alcohol $\mathbf{S - 2 7}$, which was subjected to Swern oxidation to provide ketone S-28. Removal of the acetonide and reprotection of the resulting diol as the bis-TMS ether provided compound $\mathbf{S - 3 0}$ which was subjected to Lindlar reduction to provide our intramolecuar Diels-Alder precursor, $\mathbf{5}$.

\section{Scheme S-3}
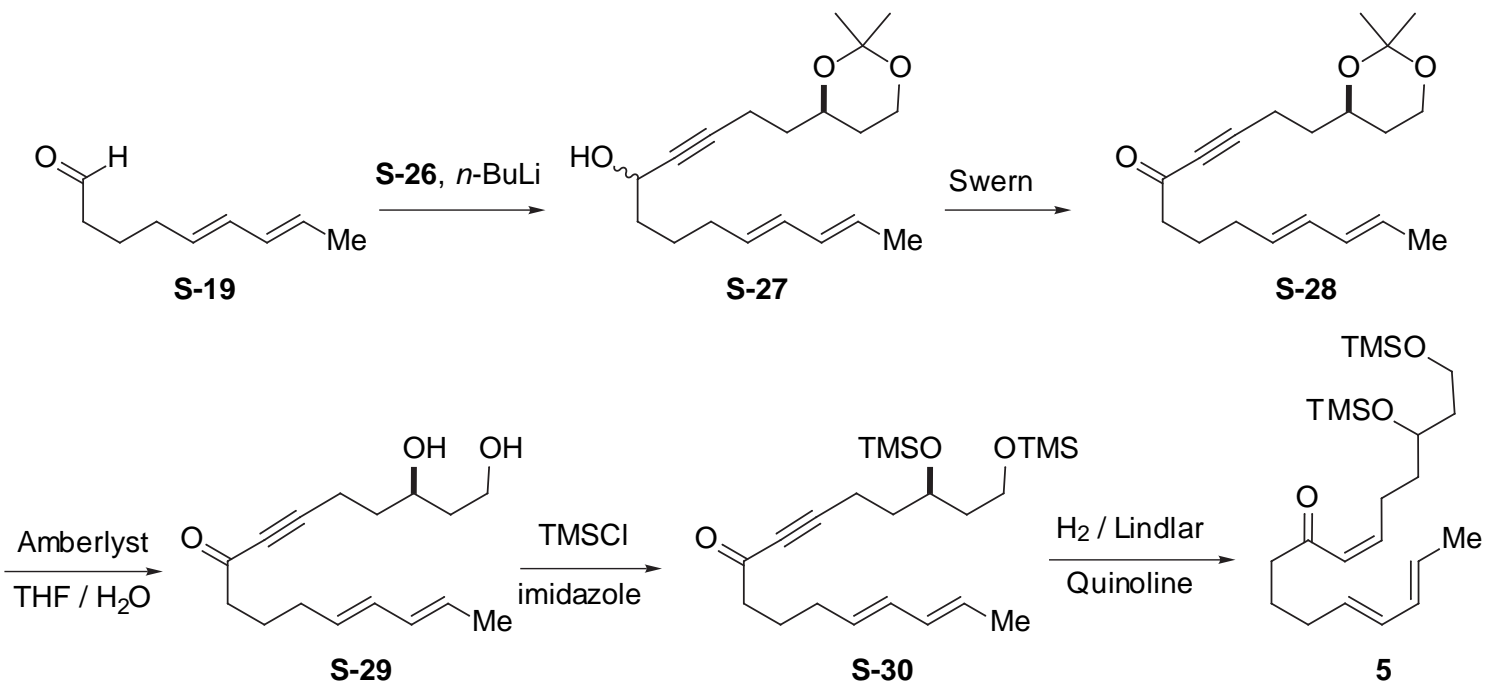

(5) Nokami, J.; Ohga, M.; Nakamoto, H.; Matsubara, T.; Hussain, I.; Kataoka, K. J. Am. Chem. Soc. 2001, 123, 9168.

(6) Rychnovsky, S. D.; Griesgraber, G.; Schlegel, R. J. Am. Chem. Soc. 1995, 117, 197.

(7) Compound $\mathbf{S - 2 4}$ is very sensitive to racemization by acid catalyzed equilibration of the acetonide, and if the hydrogenolysis is conducted with $\mathrm{Pd} / \mathrm{C}$ significant racemization occurs. See: Rychnovsky, S. D.; Griesgraber, G.; Schlegel, R. J. Am. Chem. Soc., 1995, 117, 197

(8) The use of less polar solvents, such as THF or ether, provided significant quantities of the elimination byproducts. 


\section{Materials and Methods}

All air or moisture sensitive reactions were conducted in flame-dried glassware under an atmosphere of nitrogen. Diethyl ether and tetrahydrofuran (THF) were distilled under nitrogen from sodium benzophenone ketyl. Methylene chloride, triethylamine, pyridine, N,Ndimethylformamide, and methanol were distilled under nitrogen from calcium hydride immediately prior to use. Dimethyl sulfoxide was distilled from calcium hydride under reduced pressure immediately prior to use. $p$-Toluenesulfonyl chloride was purified by dissolving in ether and washing with $10 \% \mathrm{NaOH}$ and brine, dried over $\mathrm{Na}_{2} \mathrm{SO}_{4}$, concentrated under reduced pressure and recrystallized from toluene/petroleum ether. All other reagents were purchased from Aldrich and used without further purification, except where noted otherwise. Neutral silica gel was obtained from Mallinckrodt (Mallinckrodt Silicar Silica Gel 150, 60-200 mesh (75-250 micron), pH of 5\% slurry is 7.0). ${ }^{1} \mathrm{H}$ NMR spectra were obtained at $400 \mathrm{MHz}$ or $500 \mathrm{MHz}$, and ${ }^{13} \mathrm{C} \mathrm{NMR}$ spectra were obtained at $100 \mathrm{Mhz}$ or $125 \mathrm{Mhz}$ as indicated, in chloroform- $D$ at room temperature. Chemical shifts were referenced to the residual chloroform- $H$ peak, which was set at 7.24 ppm for ${ }^{1} \mathrm{H}$ and $77.23 \mathrm{ppm}$ (center peak) for ${ }^{13} \mathrm{C}$ spectra. Infrared spectra were obtained as thin films on $\mathrm{NaCl}$ plates. Optical rotations were collected at $589 \mathrm{~nm}$ as solutions in chloroform- $D$ at room temperature using a Jasco P-1030 digital polarimeter; concentrations (c) are reported as g/100 mL. Thin layer chromatography (TLC) was performed using EM Science Silica Gel 60 F254 glass plates that were visualized under ultraviolet light and with $p$-anisaldehyde stain. High performance liquid chromatography (HPLC) was performed using an instrument equipped with a DIACEL Chemical Industries, LTD CHIRALCEL OD column (ODOOG-HC003) or an Alltech Econosil Silica 10U, $250 \mathrm{~mm}$ by $10 \mathrm{~mm}$ semi-preparative column. Combustion analyses were performed by NuMega Resonance Labs, Inc. of San Diego, CA. Exact mass was calculated on M+Na using electrospray ionization (ESI), or on the parent ion using electron impact ionization (EI).

\section{Experimental Procedures}

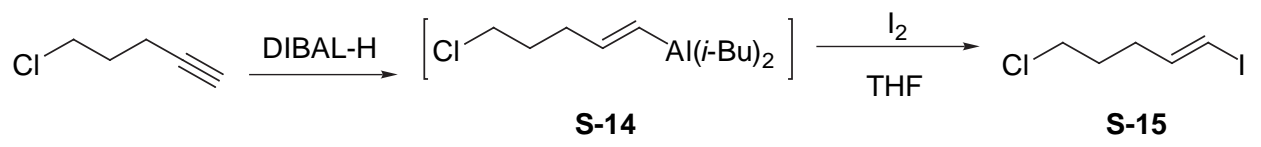

Vinyl iodide S-15': To a $1 \mathrm{~L}$ three-neck flask equipped with a condenser was added diisobutylaluminum hydride (DIBAL-H) $(247 \mathrm{~mL}$ of a 1.0M solution in hexanes, $247 \mathrm{mmol})$ and 5-chloro-1-pentyne (24.87 g, $242.5 \mathrm{mmol}$, fractionally distilled under aspirator pressure) via cannula over 30 minutes. The reaction mixture was stirred overnight at $50{ }^{\circ} \mathrm{C}$, then cooled, and the reaction flask was fitted with a distillation head. Hexanes was removed by distillation under reduced pressure. To the remaining oil was added THF $(20 \mathrm{~mL})$. The reaction was cooled to -78 ${ }^{\circ} \mathrm{C}$ and a solution of iodine $(73.9 \mathrm{~g}, 291 \mathrm{mmol})$ in THF $(150 \mathrm{~mL})$ was added via cannula over 45 minutes. The deep red-colored solution was stirred an additional 15 minutes, allowed to warm to room temperature, then quenched by cannulating the solution into a rapidly stirred mixture of hexanes $(250 \mathrm{~mL})$ and $2 \mathrm{~N} \mathrm{HCl}(250 \mathrm{~mL})$ maintained below $20^{\circ} \mathrm{C}$ by an ice bath. The resulting organic layer was washed with an additional $50 \mathrm{~mL}$ of $2 \mathrm{~N} \mathrm{HCl}$ solution. The combined aqueous layers were extracted with hexanes $(3 \times 100 \mathrm{~mL})$, and the combined organic layers were washed with saturated $\mathrm{NaHCO}_{3}(100 \mathrm{~mL})$, saturated $\mathrm{Na}_{2} \mathrm{~S}_{2} \mathrm{O}_{3}(100 \mathrm{~mL})$, and brine $(100 \mathrm{~mL})$, dried over $\mathrm{MgSO}_{4}$, and concentrated under reduced pressure. Fractional distillation under high vacuum (55$\left.53{ }^{\circ} \mathrm{C}, 0.75 \mathrm{~mm} \mathrm{Hg}\right)$ provided $44.24 \mathrm{~g}(79 \%, 192 \mathrm{mmol})$ of vinyl iodide $\mathbf{S}-\mathbf{1 5}$ as a colorless, light sensitive oil. TLC $\mathrm{R}_{\mathrm{f}}=0.56$ (10:1 hexanes/ethyl acetate). ${ }^{1} \mathrm{H}$ NMR (500 MHz) $\delta 6.46$ (dt, $J=14.3$, $7.0 \mathrm{~Hz}, 1 \mathrm{H}), 6.08(\mathrm{dq}, J=14.3,1.3 \mathrm{~Hz}, 1 \mathrm{H}), 3.52$ (t, $J=6.3 \mathrm{~Hz}, 2 \mathrm{H}), 2.21$ (ddd, $J=14.5,7.0,1.5 \mathrm{~Hz}$, 2H), $1.84(\mathrm{p}, J=7.0 \mathrm{~Hz}, 2 \mathrm{H}) .{ }^{13} \mathrm{C}$ NMR $(125 \mathrm{MHz}) \delta 144.41,76.09,43.80,32.96,30.87$. IR 3048, 2994, 2954, 2868, 2844, 1606, 1441, 1294, 1270, 1217, 944, 860, 780, 728, $650 \mathrm{~cm}^{-1}$. Exact mass calculated for $\left[\mathrm{C}_{5} \mathrm{H}_{8} \mathrm{ClI}\right]^{+}:$229.9354. Found: 229.9333 (EI). 


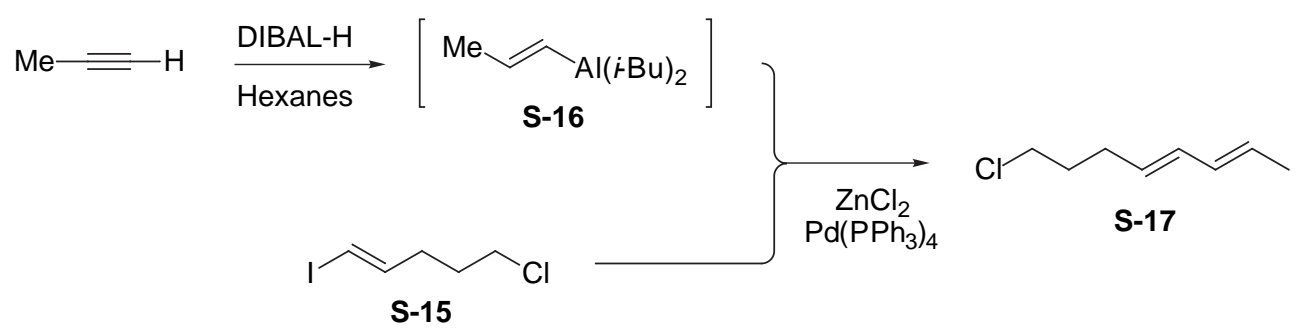

Diene S-17: ${ }^{2}$ To a large $(500 \mathrm{ml})$ Schlenk-type thick-walled glass vessel equipped with a Teflon valve was added DIBAL-H (244 $\mathrm{mL}$ of a 1.0M solution in hexanes, $244 \mathrm{mmol})$ via cannula. Propyne was collected in a graduated cylinder at $-78{ }^{\circ} \mathrm{C}(\sim 16.5 \mathrm{~mL})$ and then transferred via cannula to the rapidly stirring solution of DIBAL-H, cooled to $-78^{\circ} \mathrm{C}$. The vessel was sealed and warmed to $50{ }^{\circ} \mathrm{C}$ in a sand bath for 20 hours, then cooled to $0{ }^{\circ} \mathrm{C}$. Warning! This reaction must be conducted behind a blast shield and in a thick walled vessel with sufficient head space to accommodate the increase in pressure which accompanies warming the sealed reaction vessel to $50{ }^{\circ} \mathrm{C} !$

A separate $2 \mathrm{~L}$ flask was charged with anhydrous $\mathrm{ZnCl}_{2}(30.12 \mathrm{~g}, 221 \mathrm{mmol}$; anhydrous grade obtained from Aldrich and further dried by gentle heating under high vacuum) and THF (620 $\mathrm{mL}$ ), and a solution of vinyl iodide $\mathbf{S - 1 5}$ (44.24 g, $192 \mathrm{mmol})$ in THF (20 mL). The reaction was cooled to $0{ }^{\circ} \mathrm{C}$ and the $0{ }^{\circ} \mathrm{C}$ solution of propenyl alane $\mathbf{S}-\mathbf{1 6}$ prepared above was cannulated into the reaction mixture. Tetrakis(triphenylphosphine)palladium(0) $(0.666 \mathrm{~g}, 0.576 \mathrm{mmol})$ was then added as a solution in THF via cannula, and the sealed reaction was stirred at $0{ }^{\circ} \mathrm{C}$. Aliquots of the reaction were taken and monitored by ${ }^{1} \mathrm{H}$ NMR spectroscopy, and after 24 hours it was judged complete. The reaction was quenched by careful cannulation into a rapidly stirred mixture of pentane $(500 \mathrm{~mL})$ and $\mathrm{HCl}(250 \mathrm{~mL}$ of a $2 \mathrm{M}$ solution $)$ maintained below $15{ }^{\circ} \mathrm{C}$ by an ice bath. After addition was complete, the layers were separated and the organic layer was washed with additional $\mathrm{HCl}$ solution $(100 \mathrm{~mL}$ of a $2 \mathrm{M}$ solution). The combined aqueous layers were extracted with pentane $(3 \times 100 \mathrm{~mL})$, and the combined organic layers were washed with saturated $\mathrm{NaHCO}_{3}$ $(100 \mathrm{~mL})$, water $(2 \times 200 \mathrm{~mL})$, dried over $\mathrm{MgSO}_{4}$, filtered through a Celite pad, and concentrated under reduced pressure. The crude oil was filtered through a short plug of silica to remove any remaining palladium (eluted with pentane), then purified by fractional distillation under reduced pressure $\left(40{ }^{\circ} \mathrm{C}, 0.6 \mathrm{~mm} \mathrm{Hg}\right)$ to afford diene $\mathbf{S}-\mathbf{1 7}$ as colorless oil (18.58 g, $\left.128.5 \mathrm{mmol}, 67 \%\right)$. TLC R $\mathrm{R}_{\mathrm{f}}=0.425$ (hexanes). ${ }^{\mathrm{H}} \mathrm{H}$ NMR $\left(500 \mathrm{MHz}, \mathrm{CDCl}_{3}\right) \delta 6.06-5.97(\mathrm{~m}, 2 \mathrm{H}), 5.63-5.56(\mathrm{~m}, 1 \mathrm{H})$, 5.50-5.44 (m, 1H), $3.51(\mathrm{t}, J=6.6 \mathrm{~Hz}, 2 \mathrm{H}), 2.19(\mathrm{q}, J=7.3 \mathrm{~Hz}, 2 \mathrm{H}), 1.83(\mathrm{p}, J=6.6 \mathrm{~Hz}, 2 \mathrm{H}), 1.71(\mathrm{~d}, J$ $=6.8 \mathrm{~Hz}, 3 \mathrm{H}) .{ }^{13} \mathrm{C} \mathrm{NMR}\left(125 \mathrm{MHz}, \mathrm{CDCl}_{3}\right) \delta 131.87,131.51,129.64,127.85,44.54,32.34$, 29.76, 18.20. IR 3016, 2958, 2934, 2914, 2852, 1442, 988, 926, 727, $654 \mathrm{~cm}^{-1}$. Exact mass calculated for $\left[\mathrm{C}_{8} \mathrm{H}_{13} \mathrm{Cl}\right]^{+}:$144.0700. Found: $144.0719(\mathrm{EI})$.

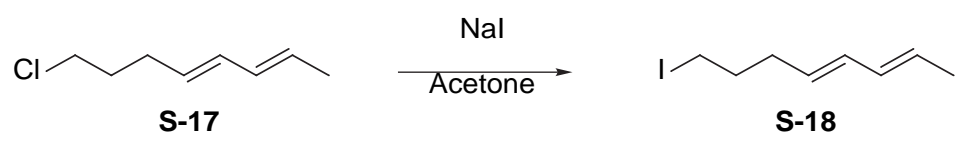

Iodide S-18: ${ }^{3}$ A $500 \mathrm{~mL}$ round bottom flask equipped with a condenser was charged with a solution of sodium iodide $(115 \mathrm{~g}, 766.7 \mathrm{mmol})$ and sodium thiosulfate $(0.1 \mathrm{~g})$ in acetone $(320$ $\mathrm{mL}$, distilled from $\mathrm{K}_{2} \mathrm{CO}_{3}$ ). Dienyl chloride $\mathbf{S}-\mathbf{1 7}(18.48 \mathrm{~g}, 127.8 \mathrm{mmol})$ was then added, and the reaction mixture was heated to reflux. After 24 hours, additional sodium iodide $(38.3 \mathrm{~g}, 255.6$ mmol) was added and the reaction was continued at reflux for an additional 24 hours, then cooled to room temperature. The reaction was diluted pentane $(200 \mathrm{~mL})$. causing the precipitation of large quantities of solids (sodium iodide and sodium chloride). The solids were filtered and washed with pentane $(300 \mathrm{~mL})$, and filterate was washed with water $(2 \times 100 \mathrm{~mL})$, and brine $(100 \mathrm{~mL})$, dried over $\mathrm{MgSO}_{4}$, filtered, and concentrated under reduced pressure. The crude colorless oil was purified by flash chromatography ( $\mathrm{SiO}_{2}$, pentane) to provide $27.54 \mathrm{~g}(116.7 \mathrm{mmol}, 91 \%)$ of iodide S-18. $T L C R_{f}=0.69\left(20: 1\right.$ hexanes/ethyl acetate). ${ }^{1} \mathrm{H} \operatorname{NMR}\left(500 \mathrm{MHz}, \mathrm{CDCl}_{3}\right) \delta 6.01(\mathrm{~m}, 2 \mathrm{H})$, 
$5.59(\mathrm{dq}, J=13.5,7.1 \mathrm{~Hz}, 1 \mathrm{H}), 5.45(\mathrm{dt}, J=14.5,6.9 \mathrm{~Hz}, 1 \mathrm{H}), 3.16(\mathrm{t}, J 6.9 \mathrm{~Hz}, 2 \mathrm{H}), 2.14(\mathrm{q}, J=$ $7.3,2 \mathrm{H}), 1.88(\mathrm{p}, J=7.1 \mathrm{~Hz}, 2 \mathrm{H}), 1.71(\mathrm{~d}, J=6.7 \mathrm{~Hz}, 3 \mathrm{H}) .{ }^{13} \mathrm{C}$ NMR $\left(125 \mathrm{MHz}, \mathrm{CDCl}_{3}\right) \delta$ $131.75,131.27,129.08,127.75,33.06,32.90,18.04,6.53$. IR 3014, 2958, 2930, 2850, 1437, 1209, $1172,987,926 \mathrm{~cm}^{-1}$.

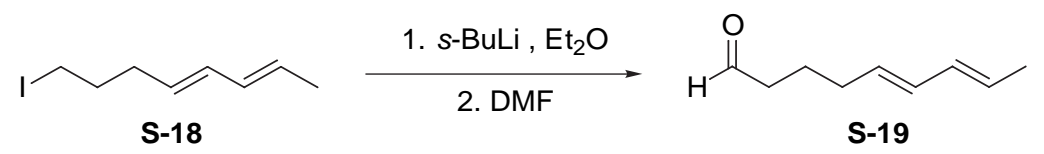

Aldehyde S-19: 4 To a $-78{ }^{\circ} \mathrm{C}$ solution of sec-butyllithium $(87.3 \mathrm{~mL}, 120.5 \mathrm{mmol}, 1.38 \mathrm{M}$ in cyclohexane) in diethyl ether $(230 \mathrm{~mL})$ was added a solution of iodide $\mathbf{S - 1 8}(12.93 \mathrm{~g}, 54.78$ mmol) in diethyl ether $(20 \mathrm{~mL})$ via cannula. The reaction was stirred for 10 minutes at $-78^{\circ} \mathrm{C}$ then placed in an ice bath and stirred for 1 hour. The reaction then cooled back to $-78{ }^{\circ} \mathrm{C}$ and $\mathrm{N}, \mathrm{N}$ dimethylformamide ( $17.0 \mathrm{~mL}, 219.1 \mathrm{mmol}$, freshly distilled for $\mathrm{CaH}_{2}$ under reduced pressure) was added and the mixture was in an ice bath for 1 hour. The reaction was quenched by the addition of $\mathrm{HCl}(50 \mathrm{~mL}, 2 \mathrm{M})$ and the organic layer was separated and washed with additional $\mathrm{HCl}(15 \mathrm{~mL})$, saturated $\mathrm{NaHCO}_{3}$ solution $(20 \mathrm{~mL})$ and brine $(20 \mathrm{~mL})$. The acidic aqueous layers were combined as were the $\mathrm{NaHCO}_{3}$ and brine layers, and each were extracted with $\mathrm{CH}_{2} \mathrm{Cl}_{2}$ until no product was detected by TLC in the organic extract $(9 \times 20 \mathrm{~mL})$. The combined organic layers were washed with brine $(20 \mathrm{~mL})$, dried over $\mathrm{MgSO}_{4}$, filtered, and concentrated under reduced pressure. The crude oil was filtered through silica gel (1:1 pentane/diethyl ether) to remove DMF and purified by flash chromatography ( $\mathrm{SiO}_{2}$, pentane/diethyl ether 40:1 to 20:1 to 10:1 to 5:1 gradient elution) to provide aldehyde $\mathbf{S}-19$ as colorless oil $(5.10 \mathrm{~g}, 36.9 \mathrm{mmol}, 67 \%)$. TLC $\mathrm{R}_{\mathrm{f}}=0.40(9: 1$ hexanes/ethyl acetate). ${ }^{1} \mathrm{H}$ NMR $\left(500 \mathrm{MHz}, \mathrm{CDCl}_{3}\right) \delta 9.74(\mathrm{t}, J=1.6 \mathrm{~Hz}, 1 \mathrm{H}), 5.99(\mathrm{~m}, 2 \mathrm{H}), 5.58$ $(\mathrm{m}, 1 \mathrm{H}), 5.47(\mathrm{~m}, 1 \mathrm{H}), 2.42(\mathrm{dt}, J=1.6,7.5 \mathrm{~Hz}, 2 \mathrm{H}), 2.08(\mathrm{q}, J=7.1 \mathrm{~Hz}, 2 \mathrm{H}), 1.71(\mathrm{~d}, J=7.1 \mathrm{~Hz}$, $3 \mathrm{H}), 1.70(\mathrm{p}, J=7.3 \mathrm{~Hz}, 2 \mathrm{H}) .{ }^{13} \mathrm{C} \mathrm{NMR}\left(125 \mathrm{MHz}, \mathrm{CDCl}_{3}\right) \delta 202.60,131.48,131.31,130.19$, 127.66, 43.16, 31.77, 21.70, 18.02. IR 3016, 2932, 2720, 1725, 1438, 989, $668 \mathrm{~cm}^{-1}$.

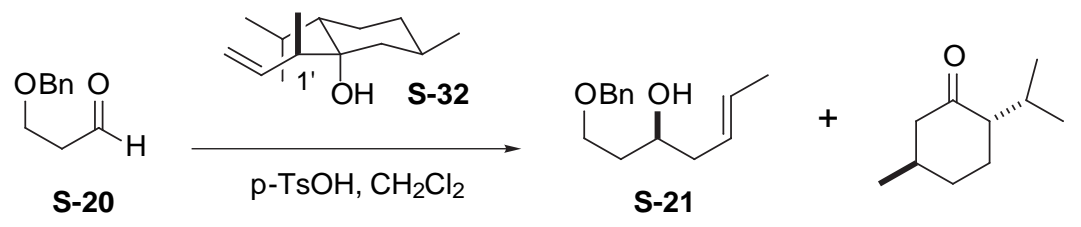

Alcohol S-21: To a solution of $\beta$-benzyloxy aldehyde S-20 ${ }^{9}$ (32.618 g, $198.65 \mathrm{mmol}$ ) (prepared by mono-protection of 1,3-propane diol with benzyl bromide and sodium hydride in THF followed by swern oxidation) in methylene chloride (1L) was added a 7:1 diastereomeric mixture (at C1') of crotyl donor $\mathbf{S - 3 2}{ }^{1}$ (50.143 g, $238.4 \mathrm{mmol}$ ) via cannula. To this solution was added $p$ toluenesulfonic acid monohydrate $(3.78 \mathrm{~g}, 19.87 \mathrm{mmol})$. The reaction was capped and stirred at room temperature for 2 days, at which point it was sky blue in color. Aldehyde consumption was monitored by ${ }^{1} \mathrm{H}$ NMR spectroscopy. The reaction was quenched by the addition of saturated $\mathrm{NaHCO}_{3}$ solution $(200 \mathrm{~mL})$ and the aqueous layer was extracted with diethyl ether $(3 \times 100 \mathrm{~mL})$. The combined organic layers were washed with brine, dried over $\mathrm{MgSO}_{4}$, filtered through Celite, and concentrated under reduced pressure. Distillation under reduced pressure $\left(90-135^{\circ} \mathrm{C} / 1.05\right.$ $\mathrm{mmHg}$ ) afforded alcohol S-21 (31.95 g, $145.0 \mathrm{mmol}, 73 \%$ yield). TLC $\mathrm{R}_{\mathrm{f}}=0.59$ (2:1 hexanes/ethyl acetate). ${ }^{1} \mathrm{H}$ NMR $(500 \mathrm{MHz}) \delta 7.30(\mathrm{~m}, 5 \mathrm{H}), 5.46(\mathrm{~m}, 2 \mathrm{H}), 4.50\left(\mathrm{AB}, J_{\mathrm{AB}}=12.3 \mathrm{~Hz}, 2 \mathrm{H}\right), 3.79$ (m, 1H), 3.59-3.70 (m, 2H), $2.78(\mathrm{~d}, J=2.7 \mathrm{~Hz}, 1 \mathrm{H}, \mathrm{OH}), 2.15(\mathrm{dd}, J=6.4,6.4,2 \mathrm{H}) 1.65(\mathrm{~d}, J=1.3$ $\mathrm{Hz}, 3 \mathrm{H}) .{ }^{13} \mathrm{C}$ NMR $(100 \mathrm{MHz}) \delta 138.21,128.63,128.54,127.90,127.86,127.36,73.48,70.79$, 69.15, 40.92, 36.08, 18.27. IR 3430 3027, 2919, 2861, 1494, 1449, 1365, 1310, 1207, $1096 \mathrm{~cm}^{-1}$. $[\alpha]_{\mathrm{D}^{20}}=-2.27(c=2.15)$. HPLC (CHIRALCEL OD column, 200:2:1 hexanes/chloroform/ethanol, $2 \mathrm{~mL} / \mathrm{min}) 98 \%$ ee. Anal. Calcd. for $\mathrm{C}_{14} \mathrm{H}_{20} \mathrm{O}_{2}$ : C, 76.33; H, 9.15. Found: C, 76.08; H, 8.76.

(9) Crimmins, M. T.; Washburn, D. G. Tetrahedron Lett. 1998, 39, 7487. 


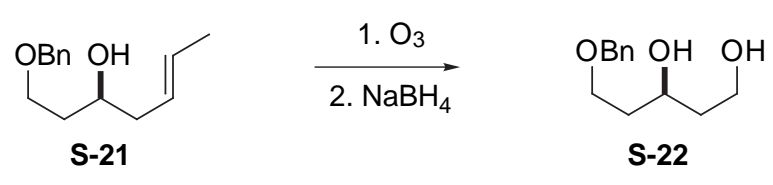

Diol S-22: 6 Ozone was bubbled through a $-78{ }^{\circ} \mathrm{C}$ solution of alcohol S-21 (55.87 $\mathrm{g}$, $253.6 \mathrm{mmol})$ and potassium carbonate $(70.1 \mathrm{~g}, 507.2 \mathrm{mmol})$ in methanol $(300 \mathrm{~mL})$ and methylene chloride $(900 \mathrm{~mL})$ for 4.5 hours. TLC indicated that the reaction was complete immediately before it turned light blue. Nitrogen was bubbled through the solution for 30 minutes to purge excess ozone. Sodium borohydride $(19.19 \mathrm{~g}, 507.2 \mathrm{mmol})$ was added, and the solution was warmed to room temperature. After stirring for 6 hours ammonium chloride (162.8 g, 3.04 mol) was added, a condenser was then placed on the flask, and the reaction mixture was stirred overnight, filtered through Celite and concentrated under reduced pressure. The resulting boronic ester was hydrolyzed by dissolving the crude foam in chloroform and $2 \mathrm{~N} \mathrm{HCl}$. The organic layer was washed with water $(5 \times 100 \mathrm{~mL})$ and the combined aqueous washes were extracted with methylene chloride $(2 \times 100 \mathrm{~mL})$. The combined organic layers were washed with saturated $\mathrm{NaHCO}_{3}$, brine, dried over $\mathrm{MgSO}_{4}$, filtered and concentrated under reduced pressure. The crude oil was purified by flash chromatography (1:1 hexanes/diethyl ether to 1:4:1 hexanes/diethyl ether/methanol) and diol S-22 (42.66 g, $202.9 \mathrm{mmol}, 80 \%$ ) was obtained as a clear oil. TLC $\mathrm{R}_{\mathrm{f}}=0.35$ (ethyl acetate). ${ }^{1} \mathrm{H}$ NMR (400 MHz) $\delta 7.30(\mathrm{~m}, 5 \mathrm{H}), 4.50(\mathrm{~s}, 2 \mathrm{H}), 4.06(\mathrm{~m}, 1 \mathrm{H}), 3.80(\mathrm{~m}, 2 \mathrm{H}), 3.71$ (A of ABXY), $\left.J_{\mathrm{AB}}=9.3 \mathrm{~Hz}, J_{\mathrm{AX}}=4.5 \mathrm{~Hz}, J_{\mathrm{AY}}=5.6 \mathrm{~Hz}, 1 \mathrm{H}\right), 3.65\left(\mathrm{~B}\right.$ of $\mathrm{ABXY}, J_{\mathrm{BA}}=9.3 \mathrm{~Hz}, J_{\mathrm{BX}}=4.1 \mathrm{~Hz}, J_{\mathrm{BY}}=8.7$ $\mathrm{Hz}, 1 \mathrm{H}), 3.26(\mathrm{~s}, 2 \mathrm{H}, \mathrm{OH}), 1.75(\mathrm{~m}, 4 \mathrm{H}) .{ }^{13} \mathrm{C} \mathrm{NMR}(100 \mathrm{MHz}) \delta 137.93,128.79,128.02,127.89$, 73.58, 71.97, 69.28, 61.66, 38.61, 36.74. IR 3376, 3064, 3032, 2937, 2869, 1450, 1364, 1208, $1089,739,698 \mathrm{~cm}^{-1} .[\alpha]_{\mathrm{D}}^{20}=-5.5(c=2.45)$. Exact mass calculated for $\left[\mathrm{C}_{12} \mathrm{H}_{18} \mathrm{O}_{3} \mathrm{Na}^{+}\right.$: 233.11481. Found: 233.11515 (ESI).

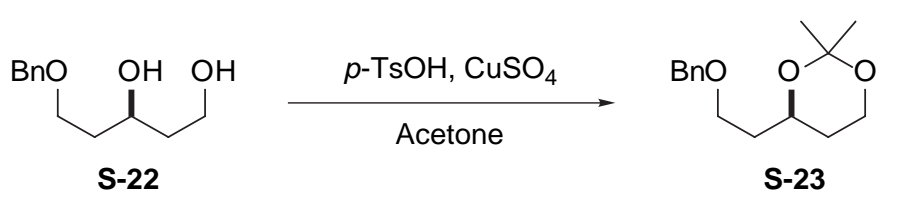

Acetonide S-23: To a solution of diol S-22 (38.92 g, $185.1 \mathrm{mmol})$ in acetone $(600 \mathrm{~mL}$, distilled under nitrogen from $\mathrm{K}_{2} \mathrm{CO}_{3}$ ) was added anhydrous copper sulfate $(88.63 \mathrm{~g}, 555.3 \mathrm{mmol})$ and $p$-toluenesulfonic acid $(7.04 \mathrm{~g}, 37.02 \mathrm{mmol})$. The solution was stirred overnight at room temperature. Additional copper sulfate $(59 \mathrm{~g}, 365.7 \mathrm{mmol}, 2$ equiv) was added and the reaction was stirred for an additional day. The acid was quenched by the addition of triethylamine $(25.8 \mathrm{~mL}$, $155.1 \mathrm{mmol}$ ) and stirred for 30 minutes. The reaction mixture was filtered through Celite, washed with acetone, and concentrated under reduced pressure. Purification by flash chromatography (hexanes to 9:1 hexanes/diethyl ether) afforded acetonide S-23 (42.90 g, $171.4 \mathrm{mmol}, 92 \%)$. TLC $\mathrm{R}_{\mathrm{f}}=0.63\left(2: 1\right.$ hexanes/ethyl acetate). ${ }^{1} \mathrm{H}$ NMR $(500 \mathrm{MHz}) \delta 7.30(\mathrm{~m}, 5 \mathrm{H}), 4.50\left(\mathrm{~A}\right.$ of $\mathrm{AB}, J_{\mathrm{AB}}=5.9$ $\mathrm{Hz}, 1 \mathrm{H}), 4.46$ (B of $\left.\mathrm{AB}, J_{\mathrm{AB}}=5.9 \mathrm{~Hz}, 1 \mathrm{H}\right) 4.05$ (tdd, $\left.J=11.6,4.8,1.6 \mathrm{~Hz}, 1 \mathrm{H}\right), 3.95$ (td, $J=12.1,2.9$ $\mathrm{Hz}, 1 \mathrm{H}), 3.80$ (ddd, $J=6.4,5.3,1.5 \mathrm{~Hz}, 1 \mathrm{H}), 3.60-3.49(\mathrm{~m}, 2 \mathrm{H}), 1.79-1.68(\mathrm{~m}, 1 \mathrm{H}), 1.43(\mathrm{~s}, 3 \mathrm{H})$, $1.42-1.14(\mathrm{~m}, 1 \mathrm{H}), 1.35(\mathrm{~s}, 3 \mathrm{H}) .{ }^{13} \mathrm{C}$ NMR $(100 \mathrm{MHz}) \delta 138.69,128.56,127.85,127.76,98.46$, 73.20, 66.29, 66.13, 60.19, 36.82, 31.52, 30.19, 19.46. IR 2940, 2866, 1453, 1374, 1244, 1194, $1102,969,740 \mathrm{~cm}^{-1}$. $[\alpha]_{\mathrm{D}}{ }^{20}=-33.094(c=2.23)$. Anal. Calcd. for $\mathrm{C}_{15} \mathrm{H}_{22} \mathrm{O}_{3}$ : C, 71.97; $\mathrm{H}, 8.86$. Found: C, 71.60; H, 8.56. 


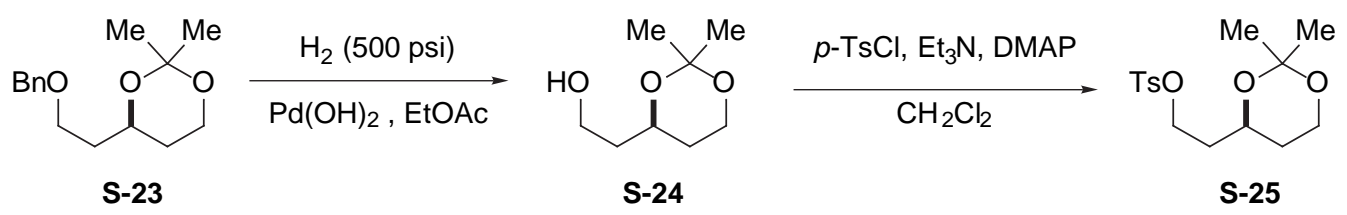

Tosylate S-25: Acetonide S-23 (3.90 g, $15.58 \mathrm{mmol})$ was dissolved in ethyl acetate (52 $\mathrm{mL}$, distilled from $\left.\mathrm{CaH}_{2}\right)$, Pearlman's catalyst $\left(\mathrm{Pd}(\mathrm{OH})_{2}\right.$ on carbon, $20 \% \mathrm{Pd}$ by wt) (109 mg) was added, and the stirred reaction mixture was subjected to hydrogen (500 psi) for 2 hours. Additional catalyst $(53 \mathrm{mg}$ ) was added and the reaction was stirred under hydrogen (500 psi) for an additional 1.5 hours. The reaction mixture was filtered through Celite, concentrated under reduced pressure, and the crude product $(\mathbf{S - 2 4})$ was imediately subjected to the next reaction.

To a solution of alcohol $\mathbf{S - 2 4}$ in $\mathrm{CH}_{2} \mathrm{Cl}_{2}(52 \mathrm{~mL})$ was added triethylamine $(6.5 \mathrm{~mL}, 46.74$ mmol), 4-dimethylaminopyridine $(95 \mathrm{mg}, 0.78 \mathrm{mmol})$, and $p$-toluenesulfonyl chloride $(4.455 \mathrm{~g}$, $23.37 \mathrm{mmol})$. The reaction mixture was stirred for one day at room temperature, then diluted with hexanes $(100 \mathrm{~mL})$ and washed with water $(5 \mathrm{~mL})$. The aqueous layer was extracted with $\mathrm{CH}_{2} \mathrm{Cl}_{2}$ $(2 \times 10 \mathrm{~mL})$ and the combined organic layers were washed with saturated $\mathrm{NaHCO}_{3}(10 \mathrm{~mL})$ and brine $(10 \mathrm{~mL})$, dried over $\mathrm{MgSO}_{4}$ and concentrated under reduced pressure. Purification by flash chromatography (10:1 hexanes/ethyl acetate) afforded $\mathbf{S}-25$ (4.115 g, 13.09 mmol, 84\% from $\mathbf{S}$ 23). TLC $\mathrm{R}_{\mathrm{f}}=0.49\left(2: 1\right.$ hexanes/ethyl acetate). ${ }^{1} \mathrm{H}$ NMR (400 MHz) $\delta 7.77(\mathrm{~d}, J=8.4 \mathrm{~Hz}, 2 \mathrm{H})$, $7.33(\mathrm{dd}, J=0.5,8.0 \mathrm{~Hz}, 2 \mathrm{H}), 4.14$ (ddd, $J=4.85,8.8,9.6 \mathrm{~Hz}, 1 \mathrm{H}), 4.05$ (td, $J=5.3,9.6 \mathrm{~Hz}, 1 \mathrm{H}), 3.97-$ $3.92(\mathrm{~m}, 1 \mathrm{H}), 3.89(\mathrm{dt}, J=2.9,12 \mathrm{~Hz}, 1 \mathrm{H}), 3.77(\mathrm{ddd}, J=1.6,5.4,12 \mathrm{~Hz}, 1 \mathrm{H}), 2.43(\mathrm{~s}, 3 \mathrm{H}), 1.80-1.68$ $(\mathrm{m}, 2 \mathrm{H}), 1.51$ (tdd, $J=5.5,12.5,12 \mathrm{~Hz}, 1 \mathrm{H}), 1.35$ (ddt, $J=2,12.5,2.5 \mathrm{~Hz}, 1 \mathrm{H}), 1.33(\mathrm{~s}, 3 \mathrm{H}), 1.27(\mathrm{~s}$, 2H). ${ }^{13} \mathrm{C}$ NMR $(100 \mathrm{MHz}) \delta 144.98,133.21,130.04,128.13,98.58,66.93,64.91,59.92,35.77$, $31.20,30.00,21.84,19.27$. IR 2950, 2876, 1599, 1454, 1363, 1181, 1121, 960, $912 \mathrm{~cm}^{-1}$. $[\alpha]_{\mathrm{D}}{ }^{20}=+22.74(c=1.41)$. HPLC (CHIRALCEL OD column, 100:1 hexanes/ethyl acetate, 2 mL/min) 94 \%ee. Anal. Calcd. for $\mathrm{C}_{15} \mathrm{H}_{22} \mathrm{O}_{5} \mathrm{~S}: \mathrm{C}, 57.30 ; \mathrm{H}, 7.05 ; \mathrm{S}, 10.20$. Found: C, 56.92; H, 7.39; S, 10.60 .
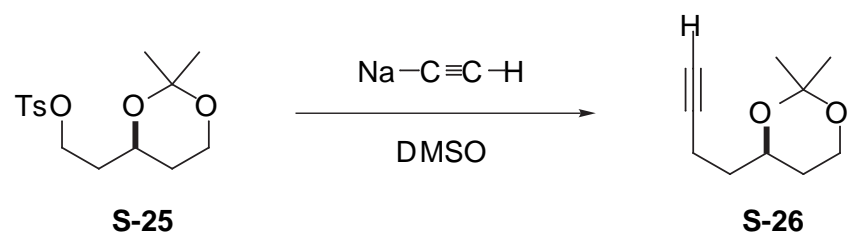

Alkyne S-26: A suspension of sodium acetylide (34 g of an $18 \mathrm{wt} \%$ suspension in mineral oil) was transferred to a pear shaped flask and washed with dry hexanes to remove mineral oil $\left(5 \times 20 \mathrm{~mL}\right.$, hexanes were distilled under $\mathrm{N}_{2}$ from $\left.\mathrm{CaH}_{2}\right)$. Traces of hexanes were removed under high vacuum pressure to provide sodium acetylide powder $(5.17 \mathrm{~g}, 107.7 \mathrm{mmol})$. This was added as a solution in DMSO $(50 \mathrm{~mL})$ via cannula to a solution of tosylate $\mathbf{S - 2 5}(19.87 \mathrm{~g}, 63.20$ $\mathrm{mmol})$ in DMSO $(250 \mathrm{~mL})$ cooled in a water bath $\left(15^{\circ} \mathrm{C}\right)$. The deep red reaction mixture was quenched after 10 minutes with saturated $\mathrm{NH}_{4} \mathrm{Cl}(40 \mathrm{~mL})$ and solid $\mathrm{NH}_{4} \mathrm{Cl}(0.5 \mathrm{~g})$. The aqueous layer was extracted with pentane $(5 \times 100 \mathrm{~mL})$ and ether $(3 \times 100 \mathrm{~mL})$ until no additional product was visible by TLC in the extract. The combined organic extracts were washed with brine, dried over $\mathrm{MgSO}_{4}$, and concentrated under reduced pressure without heating. Purification by flash chromatography (20:1 pentane/Et $\left.{ }_{2} \mathrm{O}\right)$ afforded alkyne $\mathbf{S - 2 6}(9.47 \mathrm{~g}, 56.31 \mathrm{mmol}, 89 \%)$ as a clear oil. $\mathrm{TLC} \mathrm{R}_{\mathrm{f}}=0.50$ (4:1 hexanes/ethyl acetate). ${ }^{1} \mathrm{H}$ NMR (400 MHz) $\delta$ 4.01-3.92 (m, 2H), 3.81 (ddt, $J=1.7,5.4,12 \mathrm{~Hz}, 1 \mathrm{H}), 2.33-2.19(\mathrm{~m}, 2 \mathrm{H}), 1.91(\mathrm{td}, J=1.3,2.7 \mathrm{~Hz}, 1 \mathrm{H}), 1.51-1.71(\mathrm{~m}, 3 \mathrm{H})$, 1.429 (s, 3H), $1.415(\mathrm{~m}, 1 \mathrm{H}), 1.346$ (s, 3H). ${ }^{13} \mathrm{C}$ NMR (100 MHz) $\delta$ 98.54, 84.20, 68.523, 67.34, 60.08, 35.06, 31.18, 30.11, 19.372, 14.28. IR 3293, 2986, 2944, 2870, 1440, 1376, 1258, 1198, $1110.5,973,852 \mathrm{~cm}^{-1} \cdot[\alpha]_{\mathrm{D}}^{20}=+54.79(c=1.65)$. 

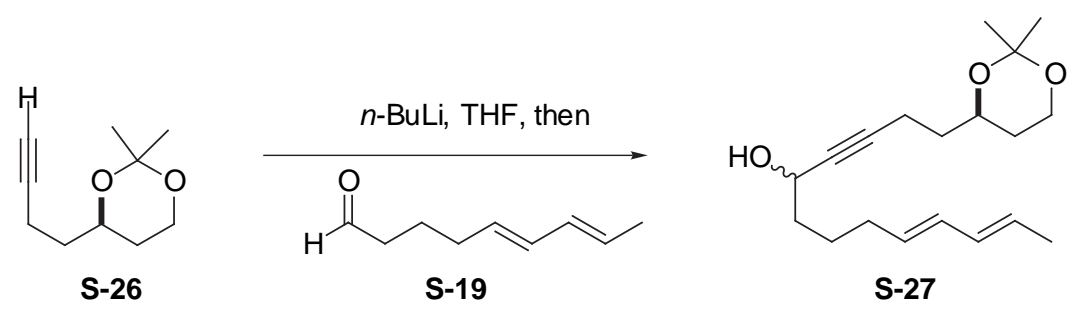

Alcohol S-27: A solution of alkyne S-26 (3.54 g, $21.02 \mathrm{mmol})$ in THF (120 mL) was cooled to $-78{ }^{\circ} \mathrm{C}$. $n$-Butyllithium $(16.3 \mathrm{~mL}, 25.23 \mathrm{mmol}, 1.55 \mathrm{M}$ in hexanes) was then added via cannula, and the reaction was stirred for $10 \mathrm{~min}$. A solution of dienyl aldehyde $\mathbf{S - 1 9}$ (3.66 g, 26.49 $\mathrm{mL}$ ) in $20 \mathrm{~mL}$ of THF was then added quickly and the reaction was stirred an additional 2 hours at $-78{ }^{\circ} \mathrm{C}$. The dry ice-acetone cold bath was removed and the reaction was allowed to slowly warm to room temperature before it was diluted with diethyl ether $(150 \mathrm{~mL})$ and quenched with saturated $\mathrm{NaHCO}_{3}$ solution $(50 \mathrm{~mL})$. The aqueous layer was extracted with diethyl ether $(3 \times 50 \mathrm{~mL})$, and the combined organic layers were washed with brine $(50 \mathrm{~mL})$, dried over $\mathrm{MgSO}_{4}$, filtered through a Celite pad, and concentrated under reduced pressure. Purification by flash chromatography (10:1 to 2:1 hexanes/ethyl acetate solvent gradient) provided $4.90 \mathrm{~g}$ (15.98 mmol, 76\%) of alcohol S-27 as a colorless oil. TLC $\mathrm{R}_{\mathrm{f}}=0.23$ (4:1 hexanes/ethyl acetate). ${ }^{1} \mathrm{H}$ NMR $\left(500 \mathrm{MHz}, \mathrm{CDCl}_{3}\right) \delta 5.99$ $(\mathrm{m}, 2 \mathrm{H}), 5.59-5.48(\mathrm{~m}, 2 \mathrm{H}), 4.32(\mathrm{br} \mathrm{q}, J=5 \mathrm{~Hz}, 1 \mathrm{H}), 3.94(\mathrm{~m}, 1 \mathrm{H}), 3.95(\mathrm{dt}, J=2.8,12 \mathrm{~Hz}, 1 \mathrm{H})$, 3.81 (ddd, $J=1.5,5.5,12.0 \mathrm{~Hz}, 1 \mathrm{H}), 2.28(\mathrm{~m}, 2 \mathrm{H}), 2.07(\mathrm{q}, J=7.0 \mathrm{~Hz}, 2 \mathrm{H}), 1.71(\mathrm{~d}, J=6.0 \mathrm{~Hz}$, $3 \mathrm{H}), 1.72-1.47(\mathrm{~m}, 8 \mathrm{H}), 1.42(\mathrm{~s}, 3 \mathrm{H}), 1.40(\mathrm{~m}, 1 \mathrm{H}), 1.35(\mathrm{~s}, 3 \mathrm{H}) .{ }^{13} \mathrm{C}$ NMR $\left(100 \mathrm{MHz}, \mathrm{CDCl}_{3}\right) \delta$ $131.79,131.39,130.92,127.21,98.51,84.94,81.63,67.47,62.62,60.03,37.78,35.26,32.28$, $31.14,30.06,25.15,19.37,18.15,14.52$. IR 3433, 3014, 2992, 2940, 2862, 1435, 1381, 1370, $1199,1161,1126,1087,988 \mathrm{~cm}^{-1} \cdot[\alpha]_{\mathrm{D}^{20}}=+33.66(c=3.92)$.

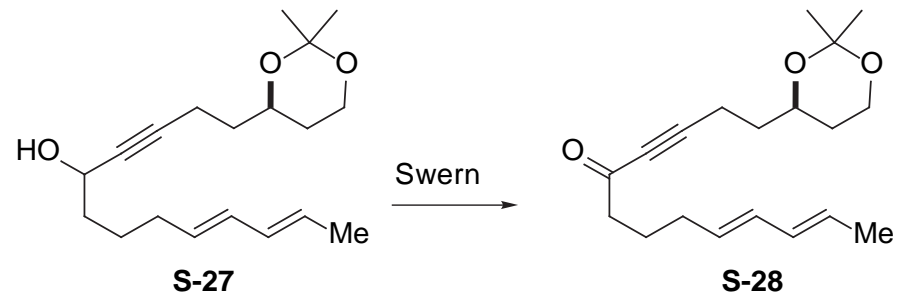

Ketone S-28: To a flask containing $\mathrm{CH}_{2} \mathrm{Cl}_{2}(200 \mathrm{~mL})$ and oxalyl chloride $(2.63 \mathrm{~mL}, 30.14$ $\mathrm{mmol}$ ) cooled to $-78{ }^{\circ} \mathrm{C}$ was added dimethyl sulfoxide $(3.56 \mathrm{~mL}, 50.22 \mathrm{mmol}$, freshly distilled from $\mathrm{NaH}$ under reduced pressure). After stirring for 30 minutes, a solution of propargyl alcohol S-27 (7.696 g, $25.11 \mathrm{mmol})$ in $\mathrm{CH}_{2} \mathrm{Cl}_{2}(50 \mathrm{~mL})$ was added dropwise over 40 minutes. The mixture was stirred for an additional 30 minutes at $-78^{\circ} \mathrm{C}$. Triethylamine $(17.5 \mathrm{~mL}, 125.6 \mathrm{mmol}$, freshly distilled from $\mathrm{CaH}_{2}$ ) was then added and the reaction was allowed to warm to room temperature. The reaction was quenched by the addition of saturated $\mathrm{NaHCO}_{3}$ solution $(15 \mathrm{~mL})$ and hexanes $(100 \mathrm{~mL})$, and the organic layer was washed with saturated $\mathrm{NaHCO}_{3}(25 \mathrm{~mL})$, then water $(20 \mathrm{~mL})$. The combined aqueous layers were extracted with hexanes $(2 \times 25 \mathrm{~mL})$, and the combined organic layers were washed with brine $(30 \mathrm{~mL})$, dried over $\mathrm{MgSO}_{4}$, filtered, and concentrated under reduced pressure. Purification by flash chromatography (gradient elution 25:1 to $5: 1$ hexanes/ethyl acetate) provided $5.17 \mathrm{~g}(17.07 \mathrm{mmol}, 68 \%)$ of ketone $\mathbf{S - 2 8}$ as a clear oil. TLC $\mathrm{R}_{\mathrm{f}}=0.43\left(4: 1\right.$ hexanes/ethyl acetate). ${ }^{1} \mathrm{H}$ NMR $\left(500 \mathrm{MHz}, \mathrm{CDCl}_{3}\right) \delta 5.98(\mathrm{~m}, 2 \mathrm{H}), 5.57(\mathrm{~m}$, $1 \mathrm{H}), 5.46(\mathrm{~m}, 1 \mathrm{H}), 3.95(\mathrm{td}, J=2.98,12.17 \mathrm{~Hz}, 1 \mathrm{H}), 3.97-3.90(\mathrm{~m}, 1 \mathrm{H}), 3.82(\mathrm{ddd}, J=1.4,5.2$, $11.7 \mathrm{~Hz}, 1 \mathrm{H}), 2.49(\mathrm{t}, J=7.3 \mathrm{~Hz}, 2 \mathrm{H}), 2.46-2.42(\mathrm{~m}, 2 \mathrm{H}), 2.05(\mathrm{q}, J=7.1 \mathrm{~Hz}, 2 \mathrm{H}), 1.75-1.64(\mathrm{~m}$, $7 \mathrm{H}), 1.57$ (ddd, $J=5.5,12.7,24.6 \mathrm{~Hz}, 1 \mathrm{H}) 1.42(\mathrm{~s}, 3 \mathrm{H}), 1.39$ (ddt, $J=1.7,12.9,2.6 \mathrm{~Hz}, 1 \mathrm{H}), 1.34$ $(\mathrm{s}, 3 \mathrm{H}) .{ }^{13} \mathrm{C}$ NMR $\left(100 \mathrm{MHz}, \mathrm{CDCl}_{3}\right) \delta 188.16,131.63,131.60,130.49,127.65,98.60,93.82$, 81.13, 67.28. 59.96, 44.97, 34.35, 31.85, 31.15, 30.08, 23.95, 19.40, 18.19, 14.86. IR 2980, 2939, $2873,2348,2211,1673,1440,1375,1197,1123,985,850,771 \mathrm{~cm}^{-1} .[\alpha]_{\mathrm{D}}^{20}=+50.90(c=5.34)$. 
Anal. Calcd. for $\mathrm{C}_{19} \mathrm{H}_{28} \mathrm{O}_{3}$ : C, 74.96; H, 9.27. Found: C 74.58; H, 9.35.
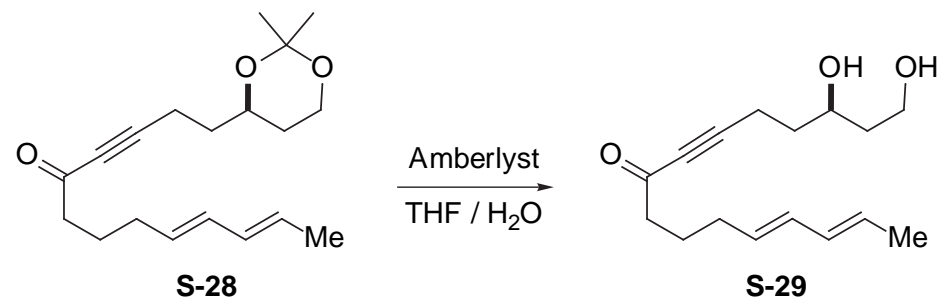

Diol S-29: A mixture of acetonide S-28 (5.1 g, $16.75 \mathrm{mmol})$, Amberlyst 15 beads $(5.1 \mathrm{~g})$, THF $(120 \mathrm{~mL})$, and water $(30 \mathrm{~mL})$ was stirred for one day at room temperature. The mixture was diluted with ethyl acetate $(100 \mathrm{~mL})$ and filtered through a Celite pad to remove the Amberlyst 15 beads. The filtrate was saturated with solid $\mathrm{NaCl}$ and the layers were separated. The aqueous layer was extracted with ethyl acetate $(3 \times 15 \mathrm{~mL})$, and the combined extracts were neutralized by the addition of solid $\mathrm{NaHCO}_{3}$ and then decanted away from the wet $\mathrm{NaHCO}_{3}$. The combined organic layers were dried over $\mathrm{MgSO}_{4}$, filtered, and concentrated under reduced pressure. Purification by flash chromatography (2:3 hexanes/ethyl acetate) provided diol S-29 (4.10 g, 15.51 mmol, 93\%) as a colorless oil. TLC $\mathrm{R}_{\mathrm{f}}=0.12$ (1:1 hexanes/ethyl acetate). ${ }^{1} \mathrm{H}$ NMR (500 MHz, $\left.\mathrm{CDCl}_{3}\right) \delta 5.98(\mathrm{~m}, 2 \mathrm{H}), 5.57(\mathrm{~m}, 1 \mathrm{H}), 5.46(\mathrm{~m}, 1 \mathrm{H}), 3.94(\mathrm{~m}, 1 \mathrm{H}), 3.88(\mathrm{~m}, 1 \mathrm{H}), 3.82(\mathrm{~m}, 1 \mathrm{H}), 3.23$ (br d, $J=3.2 \mathrm{~Hz}, 1 \mathrm{H},-\mathrm{OH}), 2.63($ br s, $1 \mathrm{H},-\mathrm{OH}), 2.50(\mathrm{t}, J=7.5 \mathrm{~Hz}, 2 \mathrm{H}), 2.49(\mathrm{t}, J=7.1 \mathrm{~Hz}, 2 \mathrm{H})$, $2.25(\mathrm{q}, J=7.1 \mathrm{~Hz}, 2 \mathrm{H}), 1.75-1.66(\mathrm{~m}, 9 \mathrm{H}) .{ }^{13} \mathrm{C} \mathrm{NMR}\left(100 \mathrm{MHz}, \mathrm{CDCl}_{3}\right) \delta 188.65,131.57$, $131.55,130.44,127.65,94.29,81.13,70.11,61.33,44.88,38.35,35.41,31.78,23.85,18.14,15.45$. IR 3335, 3008, 2927, 2350, 2208, 1660, 1430, 1359, 1235, 1060, $988 \mathrm{~cm}^{-1} \cdot[\alpha]_{\mathrm{D}}^{20}=+23.44(\mathrm{c}=$ 5.15).

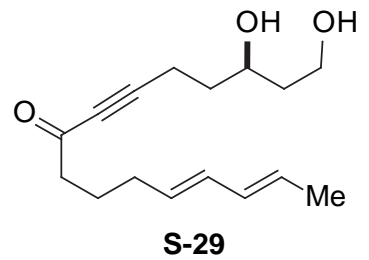

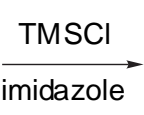

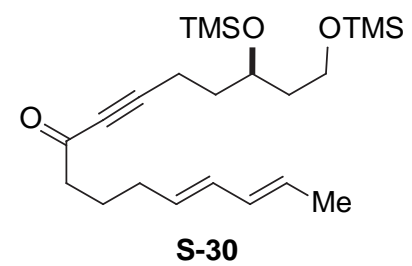

Bis-trimethylsilyether S-30: To a flask containing a solution of imidazole $(2.20 \mathrm{~g}, 32.25$ mmol) in $\mathrm{CH}_{2} \mathrm{Cl}_{2}(100 \mathrm{~mL})$ was added diol S-29 $(2.84 \mathrm{~g}, 10.75 \mathrm{mmol})$ as a solution in $\mathrm{CH}_{2} \mathrm{Cl}_{2}(10$ $\mathrm{mL})$. The reaction was placed in a room temperature water bath, and chlorotrimethylsilane $(3.4 \mathrm{~mL}$, $26.9 \mathrm{mmol}$; freshly distilled from $\mathrm{CaH}_{2}$ ) was added via syringe. The reaction was stirred at room temperature for 80 minutes then diluted with pentane $(100 \mathrm{~mL})$ and quenched with water $(10 \mathrm{~mL})$. The organic layer was separated and washed with water $(10 \mathrm{~mL})$ and brine $(10 \mathrm{~mL})$. The combined aqueous layers were extracted with pentane $(3 \times 20 \mathrm{~mL})$, and the combined organic layers were washed with brine $(10 \mathrm{~mL})$, dried over $\mathrm{MgSO}_{4}$, filtered through Celite, and concentrated under reduced pressure. Purification by flash chromatography using neutral silica gel (Mallinckrodt Silicar Silica Gel, 50:1 hexanes/ethyl acetate) afforded $3.48 \mathrm{~g}$ (8.51 mmol, 79\%) of (Bistrimethylsilyether S-30. Note that this compound undergoes partial desilylation using ordinary silica gel or triethylamine-deactivated silica gel. TLC $\mathrm{R}_{\mathrm{f}}=0.42\left(9: 1\right.$ hexanes/ethyl acetate). ${ }^{1} \mathrm{H}$ $\operatorname{NMR}\left(500 \mathrm{MHz}, \mathrm{CDCl}_{3}\right) \delta 5.98(\mathrm{~m}, 2 \mathrm{H}), 5.57(\mathrm{~m}, 1 \mathrm{H}), 5.46(\mathrm{~m}, 1 \mathrm{H}), 3.88(\mathrm{ddt}, J=4.4,7.7,5.9$ $\mathrm{Hz}, 1 \mathrm{H}), 3.60(\mathrm{t}, J=6.5 \mathrm{~Hz}, 2 \mathrm{H}), 2.50(\mathrm{t}, J=7.5 \mathrm{~Hz}, 2 \mathrm{H}), 2.39(\mathrm{~m}, 2 \mathrm{H}), 2.06(\mathrm{q}, J=7.1 \mathrm{~Hz}, 2 \mathrm{H})$, $1.75-1.60(\mathrm{~m}, 9 \mathrm{H}), 0.11(\mathrm{~s}, 9 \mathrm{H}), 0.08(\mathrm{~s}, 9 \mathrm{H}) .{ }^{13} \mathrm{C} \mathrm{NMR}\left(100 \mathrm{MHz}, \mathrm{CDCl}_{3}\right)$ _ 188.20, 131.63, 131.57, 130.55, 127.66, 94.17, 81.16, 68.23, 59.09, 44.97, 40.20, 35.48, 31.87, 23.89, 18.22, 15.36, $0.54,-0.30$. IR 3004, 2948, 2211, 1675, 1433, 1251, 1096, 1034, 988, 843, $751 \mathrm{~cm}^{-1} \cdot[\alpha]_{\mathrm{D}^{20}=}$ $+12.02(\mathrm{c}=2.23)$. Anal. Calcd. for $\mathrm{C}_{22} \mathrm{H}_{40} \mathrm{O}_{3} \mathrm{Si}_{2}: \mathrm{C}, 64.65 ; \mathrm{H}, 9.86$. Found: $\mathrm{C}$ 64.44; H, 9.65. 

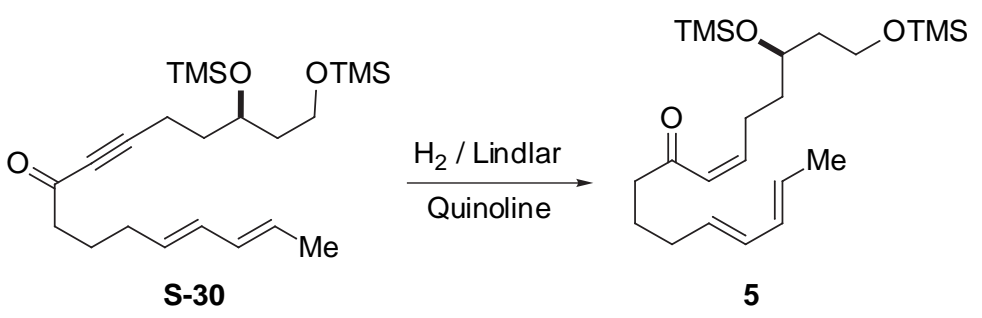

Diels-Alder precursor 5: To a three-neck round bottom flask under nitrogen was added bis(silylether) S-30 (1.50 g, $3.67 \mathrm{mmol})$, hexanes $\left(37 \mathrm{~mL}\right.$, freshly distilled from $\left.\mathrm{CaH}_{2}\right)$, quinoline $(150 \mu \mathrm{L}$, freshly distilled under high vacuum from zinc dust) and Lindlar's catalyst (5\% palladium on calcium carbonate, poisoned with lead, $0.150 \mathrm{~g}$ ). The flask was connected to an apparatus for measuring the consumption of hydrogen gas The reaction was cooled to $-78{ }^{\circ} \mathrm{C}$, and the atmosphere in the flask was replaced with $\mathrm{H}_{2}$ by briefly evacuating and filling with hydrogen several times. The reaction flask was warmed to room temperature and then stirred vigorously while the hydrogen uptake was monitored. After about 30 minutes, 1 equivalent of hydrogen had been consumed (100 mL at $27 \mathrm{~mL} / \mathrm{mmol})$, and TLC indicated that the starting material had been consumed (starting material TLC $\mathrm{R}_{\mathrm{f}}=0.59$, desired product $\mathrm{R}_{\mathrm{f}}=0.67$ in 5:1 hexanes/ethyl acetate). The mixture was filtered through a Celite pad to remove the catalyst, and the filtrate was concentrated under reduced pressure to an oil. Purified by flash chromatography using neutral silica gel (Mallinckrodt Silicar Silica Gel, 50:1 hexanes/ethyl acetate) provided Diels-Alder precursor $5(1.28 \mathrm{~g}, 3.12 \mathrm{mmol}, 85 \%)$ as a colorless oil. Note that this material in unstable to ordinary silica gel or triethylamine-deactivated silica gel. TLC $\mathrm{R}_{\mathrm{f}}=0.67$ (5:1 hexanes/ethyl acetate). ${ }^{1} \mathrm{H}$ NMR $\left(500 \mathrm{MHz}, \mathrm{CDCl}_{3}\right) \delta 6.11-5.95(\mathrm{~m}, 4 \mathrm{H}), 5.56(\mathrm{~m}, 1 \mathrm{H}), 5.48(\mathrm{~m}, 1 \mathrm{H}), 3.80$ $(\mathrm{ddd}, J=5.4,6.8,11.9 \mathrm{~Hz}, 1 \mathrm{H}), 3.61(\mathrm{t}, J=6.7 \mathrm{~Hz}, 2 \mathrm{H}), 2.63(\mathrm{q}, J=7.7 \mathrm{~Hz}, 2 \mathrm{H}), 2.42(\mathrm{t}, J=7.3$ $\mathrm{Hz}, 2 \mathrm{H}), 2.05(\mathrm{q}, J=7.1 \mathrm{~Hz}, 2 \mathrm{H}), 1.71(\mathrm{~d}, J=6.2 \mathrm{~Hz}, 3 \mathrm{H}), 1.68-1.60(\mathrm{~m}, 4 \mathrm{H}), 1.56-1.51(\mathrm{~m}$, $2 \mathrm{H}), 0.10(\mathrm{~s}, 9 \mathrm{H}), 0.08(\mathrm{~s}, 9 \mathrm{H}) .{ }^{13} \mathrm{C} \mathrm{NMR}\left(100 \mathrm{MHz}, \mathrm{CDCl}_{3}\right) \delta 201.64,148.41,131.72,131.29$, $131.10,127.47,126.87,69.33,59.39,43.67,40.34,37.25,32.12,26.19,23.79,18.24,0.60,-0.26$. IR 3005, 2945, 1692, 1618, 1429, 1415, 1252, 1097, 1043, 990, 845, 750, $680 \mathrm{~cm}^{-1} \cdot[\alpha]_{\mathrm{D}}^{20}=-2.85$ $(c=2.41)$.
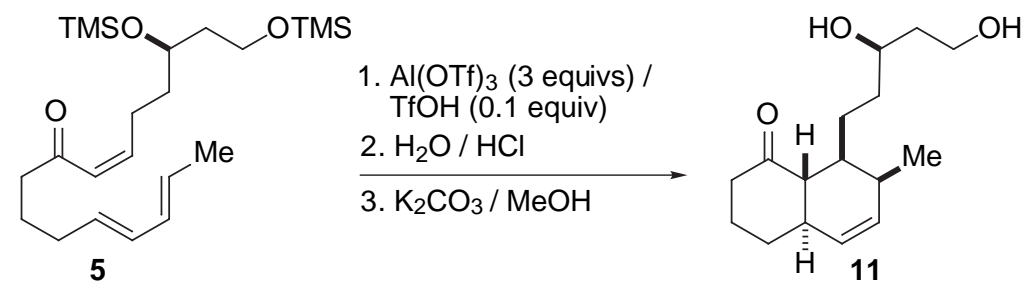

Diels-Alder adduct 11: Aluminum trifluoromethanesulfonate $(4.62 \mathrm{~g}, 9.74 \mathrm{mmol}$, powdered) was transferred in a glove box to a Schlenk-type high-pressure vessel equipped with a Teflon valve and heated gently under high vacuum. Freshly distilled $\mathrm{CH}_{2} \mathrm{Cl}_{2}(80 \mathrm{~mL})$ was added via cannula, and the resulting suspension was sonicated for 45 minutes to increase the amount of dissolved $\mathrm{Al}(\mathrm{OTf})_{3}$. The suspension was cooled to $-78{ }^{\circ} \mathrm{C}$ and a solution of Diels-Alder precursor $5(2.054 \mathrm{~g}, 5.00 \mathrm{mmol})$ in $\mathrm{CH}_{2} \mathrm{Cl}_{2}(20 \mathrm{~mL})$ was added dropwise via cannula. To the yellow reaction was added triflic acid $(0.35 \mathrm{~mL}$ of a $0.1 \mathrm{M}$ solution $(44.2 \mu \mathrm{L}$ of triflic acid in $5 \mathrm{~mL}$ $\mathrm{CH}_{2} \mathrm{Cl}_{2}$ ). The reaction vessel was sealed and placed in $-20^{\circ} \mathrm{C}$ freezer for 72 hours. Additional $\mathrm{Al}(\mathrm{OTf})_{3}(2.5 \mathrm{mmol}$, prepared by the addition of triflic acid $(0.62 \mathrm{~mL}, 7.0 \mathrm{mmol})$ to trimethyl aluminum $(1.25 \mathrm{~mL}$ of a $2.0 \mathrm{M}$ solution in hexanes, $2.50 \mathrm{mmol})$ ) was added after 16 hours and 32 hours. After three days at $-20^{\circ} \mathrm{C}$, the reaction was cooled to $-78^{\circ} \mathrm{C}$, and quenched by the addition of saturated $\mathrm{NaHCO}_{3}(300 \mathrm{~mL})$, and warmed to room temperature, and allowed to stir vigorously for one hour. The layers were separated and the aqueous layer was extracted with $\mathrm{CH}_{2} \mathrm{Cl}_{2}$ until the extract contained no product by TLC $(9 \times 100 \mathrm{~mL})$. The combined organic layers were dried over $\mathrm{MgSO}_{4}$, filtered through Celite, and concentrated under reduced pressure to afford $1.55 \mathrm{~g}$ of a 
yellow oil. This material was taken up in THF $(40 \mathrm{~mL})$ and $\mathrm{HCl}(10 \mathrm{~mL}, 2 \mathrm{M})$ and warmed to 45 ${ }^{\circ} \mathrm{C}$ overnight at which point solid sodium chloride was added to produce a biphasic mixture of brine and THF. The layers were separated, and the aqueous layer extracted with $\mathrm{Et}_{2} \mathrm{O}(3 \times 10 \mathrm{~mL})$. The organic layers were combined and dried over $\mathrm{MgSO}_{4}$, filtered through Celite, and concentrated under reduced pressure to a yellow oil. The crude oil was then dissolved in methanol $(50 \mathrm{~mL}$, freshly distilled) containing $2.0 \mathrm{~g}$ of solid $\mathrm{K}_{2} \mathrm{CO}_{3}$ and stirred at room temperature for 2 hours. The reaction was then diluted with ether and filtered through a Celite pad, concentrated under reduced pressure, and purified by flash chromatography $\left(\mathrm{SiO}_{2}, 25: 1 \mathrm{CH}_{2} \mathrm{Cl}_{2} / \mathrm{MeOH}\right)$ to provide DielsAlder adduct $11(0.665 \mathrm{~g}, 2.50 \mathrm{mmol}, 49 \%)$ as a yellow oil. This reaction was originally conducted on the enantiomer of $\mathbf{5}$, and the product, ent-11, was recrystallized from hexanes/diethyl ether and provided crystals suitable for X-ray diffraction. TLC $\mathrm{R}_{\mathrm{f}}=0.39$ (ethyl acetate). melting point $=$ $82.5-83{ }^{\circ} \mathrm{C} .{ }^{1} \mathrm{H}$ NMR $\left(500 \mathrm{MHz}, \mathrm{CDCl}_{3}\right) \delta 5.66(\mathrm{ddd}, J=2.6,4.9,9.9 \mathrm{~Hz}, 1 \mathrm{H}), 5.41(\mathrm{dt}, J=9.9$, $1.6 \mathrm{~Hz}, 1 \mathrm{H}), 3.98-3.93(\mathrm{~m}, 1 \mathrm{H}), 3.87-3.80(\mathrm{~m}, 2 \mathrm{H}), 3.26$ (br s, $1 \mathrm{H},-\mathrm{OH}), 2.78$ (br s, $1 \mathrm{H},-\mathrm{OH}), 2.43$ $(\mathrm{ddq}, J=0.9,12.0,6.5 \mathrm{~Hz}, 1 \mathrm{H}), 2.34-2.27(\mathrm{~m}, 3 \mathrm{H}), 2.16-2.05(\mathrm{~m}, 2 \mathrm{H}), 1.97-1.83(\mathrm{~m}, 3 \mathrm{H}), 1.78$ - $1.67(\mathrm{~m}, 3 \mathrm{H}), 1.54-1.45(\mathrm{~m}, 2 \mathrm{H}), 1.43-1.36(\mathrm{~m}, 1 \mathrm{H}), 1.23-1.14(\mathrm{~m}, 1 \mathrm{H}), 0.87(\mathrm{~d}, J=7.2 \mathrm{~Hz}$, $3 \mathrm{H}) .{ }^{13} \mathrm{C} \mathrm{NMR}\left(125 \mathrm{MHz}, \mathrm{CDCl}_{3}\right) \delta 214.82,133.57,128.18,71.37,62.17,52.92,46.57,43.71$, 38.57, 34.19, 33.79, 33.04, 30.15, 28.60, 23.34, 16.23. IR 3389, 2929, 2880, 2355, 1702, 1445, 1378, 1316, 1059, $730 \mathrm{~cm}^{-1}$. $[\alpha]_{\mathrm{D}}{ }^{20}=+126.2(\mathrm{c}=2.14)$. Anal. Calcd. for $\mathrm{C}_{16} \mathrm{H}_{26} \mathrm{O}_{3}: \mathrm{C}, 72.14 ; \mathrm{H}$, 9.84. Found: C 72.34; H, 9.86.
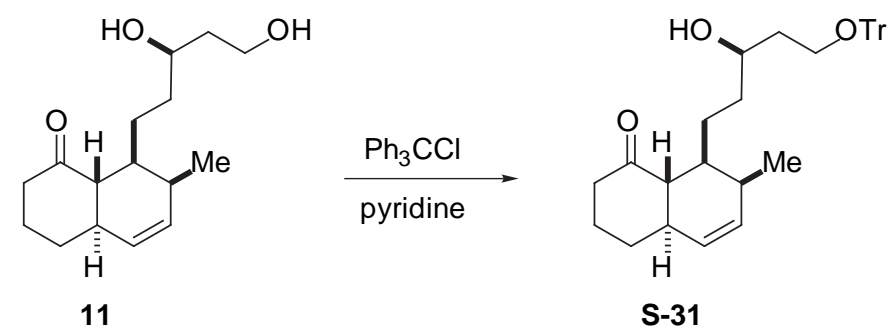

Trityl ether S-31: To a solution of diol $11(0.409 \mathrm{~g}, 1.54 \mathrm{mmol})$ in pyridine $(3 \mathrm{~mL}$, distilled from $\left.\mathrm{CaH}_{2}\right)$ at room temperature was added triphenylmethyl chloride $(1.28 \mathrm{~g}, 4.61 \mathrm{mmol}$, recrystallized from petroleum ether) in pyridine $(5 \mathrm{~mL})$ dropwise via cannula. The reaction was stirred for 3 hours at room temperature, then quenched with methanol ( $8 \mathrm{~mL}$, distilled from $\left.\mathrm{K}_{2} \mathrm{CO}_{3}\right)$ and stirred for an additional 4 hours. The reaction mixture was concentrated under reduced pressure and taken up in hexanes. Triphenylmethyl methylether crystallized and was removed from the crude solution, and the product was purified by flash chromatography $\left(\mathrm{SiO}_{2}, 20: 1\right.$ hexanes/ethyl acetate) to give trityl ether $\mathbf{S}-31(0.659 \mathrm{~g}, 1.30 \mathrm{mmol}, 84 \%)$. TLC $\mathrm{R}_{\mathrm{f}}=0.47$ (5:1 hexanes/ethyl acetate). ${ }^{1} \mathrm{H}$ NMR $\left(500 \mathrm{MHz}, \mathrm{CDCl}_{3}\right) \delta 7.42$ - $7.40(\mathrm{~m}, 6 \mathrm{H}), 7.30$ - $7.26(\mathrm{~m}, 6 \mathrm{H}), 7.23$ - $7.19(\mathrm{~m}$, $3 \mathrm{H}), 5.65(\mathrm{ddd}, J=2.8,5.2,9.9 \mathrm{~Hz}, 1 \mathrm{H}), 5.40(\mathrm{dt}, J=9.9,1.4 \mathrm{~Hz}, 1 \mathrm{H}), 3.77(\mathrm{~m}, 1 \mathrm{H}), 3.33(\mathrm{dt}, J=$ 9.3, $5.5 \mathrm{~Hz}, 1 \mathrm{H}), 3.21$ (ddd, $J=5.0,7.9,9.3 \mathrm{~Hz}, 1 \mathrm{H}), 3.03(\mathrm{br} \mathrm{d}, J=3.0 \mathrm{~Hz}, 1 \mathrm{H},-\mathrm{OH}), 2.40(\mathrm{dt}, J=$ $6.5,12.3 \mathrm{~Hz}, 1 \mathrm{H}), 2.30-2.23(\mathrm{~m}, 3 \mathrm{H}), 2.13-2.04(\mathrm{~m}, 2 \mathrm{H}), 1.99-1.92(\mathrm{~m}, 1 \mathrm{H}), 1.91$ - $1.82(\mathrm{~m}$, $2 \mathrm{H}), 1.81-1.67(\mathrm{~m}, 3 \mathrm{H}), 1.52-1.40(\mathrm{~m}, 2 \mathrm{H}), 1.33-1.26(\mathrm{~m}, 1 \mathrm{H}), 1.18-1.09(\mathrm{~m}, 1 \mathrm{H}), 0.85(\mathrm{~d}, J=$ $7.1 \mathrm{~Hz}, 3 \mathrm{H}) .{ }^{13} \mathrm{C}$ NMR $\left(100 \mathrm{MHz}, \mathrm{CDCl}_{3}\right) \delta 213.93,144.14,133.72,128.80,128.26,128.08$, 127.22, 87.36, 71.28, 62.75, 52.99, 46.55, 43.80, 37.10, 34.91, 34.33, 33.18, 30.28, 28.60, 24.25, 16.04. IR $\left(\mathrm{cm}^{-1}\right) 3504,3020,2927,2877,1707,1448,1072,911,709,634 .[\alpha]_{\mathrm{D}}{ }^{20}=+121.2(\mathrm{c}=$ 0.80). Anal. Calcd. for $\mathrm{C}_{35} \mathrm{H}_{40} \mathrm{O}_{3}$ : C, 82.64; H, 7.93. Found: $\mathrm{C}, 82.73 ; \mathrm{H}, 8.25$. 


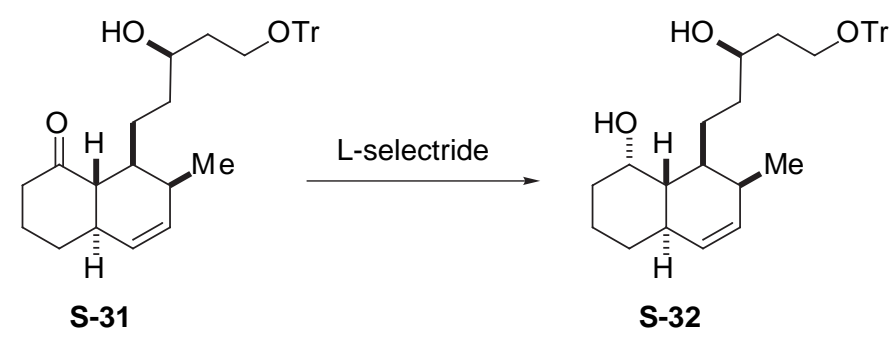

Diol S-32: A solution of S-31 (0.559 g, $1.10 \mathrm{mmol})$ in THF (3.5 mL) was cooled to -78 ${ }^{\circ} \mathrm{C}$. L-Selectride ( $3.3 \mathrm{~mL}, 3.3 \mathrm{mmol}$ of a $1.0 \mathrm{M}$ solution in THF) was then added and the reaction was stirred for one hour, then allowed to warm to room temperature and stirred an additional hour. The reaction was then cooled to $0{ }^{\circ} \mathrm{C}$ and quenched by the addition of solid $\mathrm{NaHCO}_{3}(0.924 \mathrm{~g}, 11$ mmol $)$ and $\mathrm{H}_{2} \mathrm{O}_{2}(1.25 \mathrm{~mL}$, of a $30 \%$ solution, $11 \mathrm{mmol})$ which was added dropwise. After stirring for one hour, sodium thiosulfate solution $(5 \mathrm{~mL})$ was added to dissolve the precipitates which were present in the solution, and the aqueous layer was extracted with ethyl acetate $(3 \times 5 \mathrm{~mL})$. The combined organic layers were washed with brine $(5 \mathrm{~mL})$, dried over $\mathrm{MgSO}_{4}$, filtered through Celite, and concentrated under reduced pressure. The product was purified by flash chromatography $\left(\mathrm{SiO}_{2}, 7: 1\right.$ hexanes/ethyl acetate $)$ to provide diol $\mathbf{S - 3 2}(0.539 \mathrm{~g}, 1.06 \mathrm{mmol}, 96 \%)$. TLC $\mathrm{R}_{\mathrm{f}}=0.63$ (1:1 hexanes/ethyl acetate). ${ }^{1} \mathrm{H}$ NMR $\left(400 \mathrm{MHz} \mathrm{CDCl}_{3}\right) \delta 7.42-7.40(\mathrm{~m}, 6 \mathrm{H}), 7.31-7.26(\mathrm{~m}$, $6 \mathrm{H}), 7.24-7.20(\mathrm{~m}, 3 \mathrm{H}), 5.54(\mathrm{ddd}, J=2.6,4.6,9.7 \mathrm{~Hz}, 1 \mathrm{H}), 5.37(\mathrm{~d}, J=9.7 \mathrm{~Hz}, 1 \mathrm{H}), 4.14(\mathrm{~s}, 1 \mathrm{H})$, 3.80 (tt, $J=8.6,3.3 \mathrm{~Hz}, 1 \mathrm{H}), 3.36$ (ddd, $J=5.1,9.7,14.5 \mathrm{~Hz}, 1 \mathrm{H}), 3.20(\mathrm{td}, J=4.3,8.7 \mathrm{~Hz}, 1 \mathrm{H})$, 3.13 (br s, $1 \mathrm{H},-\mathrm{OH}), 2.27-2.15(\mathrm{~m}, 2 \mathrm{H}), 1.87-1.60(\mathrm{~m}, 7 \mathrm{H}), 1.55-1.39(\mathrm{~m}, 3 \mathrm{H}), 1.34-1.16(\mathrm{~m}$, $3 \mathrm{H}), 1.07(\mathrm{td}, J=10.7,1.3 \mathrm{~Hz}, 1 \mathrm{H}), 0.98(\mathrm{qd}, J=12.4,3.3 \mathrm{~Hz}, 1 \mathrm{H}), 0.80(\mathrm{~d}, J=7.0 \mathrm{~Hz}, 3 \mathrm{H}) .{ }^{13} \mathrm{C}$ NMR $\left(100 \mathrm{MHz}, \mathrm{CDCl}_{3}\right) \delta 144.01,132.44,131.51,128.75,128.12,127.28,87.52,71.00$, $66.04,62.99,42.78, \quad 37.22,36.86,35.86,33.96,33.94,33.35,31.65,22.76,20.68,15.23$. _IR $3351,3051,3012,2926,1491,1447,1213,1073,910,710,634 \mathrm{~cm}^{-1} .[\alpha]_{\mathrm{D}}^{20}=+56.90(c=2.60)$. Anal. Calcd. for $\mathrm{C}_{35} \mathrm{H}_{42} \mathrm{O}_{3}$ : C, 82.31; $\mathrm{H}, 8.29$. Found: $\mathrm{C}, 81.93 ; \mathrm{H}, 7.94$.

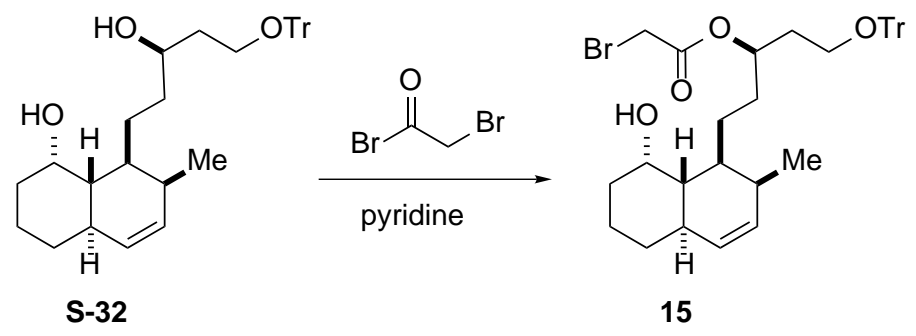

Bromoester 15: To a $-78{ }^{\circ} \mathrm{C}$ solution of $\mathbf{S - 3 2}(0.299 \mathrm{~g}, 0.585 \mathrm{mmol})$ in $\mathrm{CH}_{2} \mathrm{Cl}_{2}(10 \mathrm{~mL})$ was added pyridine $(189 \mu \mathrm{L}, 2.34 \mathrm{mmol})$. Bromoacetyl bromide $\left(102 \mu \mathrm{L}, 1.17 \mathrm{mmol}\right.$ in $\mathrm{CH}_{2} \mathrm{Cl}_{2}(2$ $\mathrm{mL})$ ) was then added via cannula over 5 minutes, and the reaction was stirred for 30 minutes, then quenched by the addition of methanol $(190 \mu \mathrm{L}, 4.68 \mathrm{mmol})$. The reaction mixture was stirred for an additional 10 minutes at $-78^{\circ} \mathrm{C}$ after which it became clear, then was allowed to warm to room temperature, diluted with hexanes, and washed with water $(2 \mathrm{~mL})$. The aqueous layer was extracted with hexanes $(3 \times 5 \mathrm{~mL})$ and the combined organic layers were washed with brine $(5 \mathrm{~mL})$, dried over $\mathrm{MgSO}_{4}$, filtered through Celite, and concentrated under reduced pressure. Flash chromatography $\left(\mathrm{SiO}_{2}, 10: 1\right.$ hexanes/ethyl acetate) provided bromoester $15(0.326 \mathrm{~g}, 0.515 \mathrm{mmol}$, $88 \%)$. TLC $\mathrm{R}_{\mathrm{f}}=0.65\left(2: 1\right.$ hexanes/ethyl acetate). ${ }^{1} \mathrm{H} \mathrm{NMR}\left(400 \mathrm{MHz}, \mathrm{CDCl}_{3}\right) \delta 7.41-7.37(\mathrm{~m}$, $6 \mathrm{H}), 7.30-7.25(\mathrm{~m}, 6 \mathrm{H}), 7.23-7.19(\mathrm{~m}, 3 \mathrm{H}), 5.52(\mathrm{ddd}, J=2.6,4.6,9.7 \mathrm{~Hz}, 1 \mathrm{H}), 5.36(\mathrm{~d}, J=9.7$ $\mathrm{Hz}, 1 \mathrm{H}), 5.20-5.14(\mathrm{~m}, 1 \mathrm{H}), 4.06(\mathrm{~s}, 1 \mathrm{H}), 3.66\left(\mathrm{H}_{\mathrm{a}}\right.$ of $\left.\mathrm{AB}, J=12.2,1 \mathrm{H}\right), 3.63\left(\mathrm{H}_{\mathrm{b}}\right.$ of $\mathrm{AB}, J=12.2$, $1 \mathrm{H}), 3.10$ (ddd, $J=5.6,9.4,20.6 \mathrm{~Hz}, 2 \mathrm{H}), 2.24-2.14(\mathrm{~m}, 2 \mathrm{H}), 1.95-1.81(\mathrm{~m}, 3 \mathrm{H}), 1.74-1.59(\mathrm{~m}, 3 \mathrm{H})$, 1.54-1.39 (m, 5H), $1.31(\mathrm{~s}, 1 \mathrm{H}), 1.13-0.91(\mathrm{~m}, 3 \mathrm{H}), 0.76(\mathrm{~d}, J=7.0 \mathrm{~Hz}, 3 \mathrm{H}) .{ }^{13} \mathrm{C} \mathrm{NMR}(100 \mathrm{MHz}$, $\left.\mathrm{CDCl}_{3}\right) \delta 167.31,144.21,132.16,131.52,128.86,128.00,127.17,86.81,74.33,65.91,59.78$, 
42.86, 36.92, 35.71, 34.80, 34.11, 33.36, 31.50, 26.44, 22.96, 20.55, 15.04. IR 3557, 348, 3012, $2925,1730,1446,1379,1283,1211,1165,1075,908,712,636 \mathrm{~cm}^{-1} .[\alpha]_{\mathrm{D}}^{20}=+34.55(c=0.67)$. Exact mass calculated for $\left[\mathrm{C}_{37} \mathrm{H}_{43} \mathrm{BrO}_{4} \mathrm{Na}\right]^{+}: 653.22369$. Found: 653.22576 (ESI).

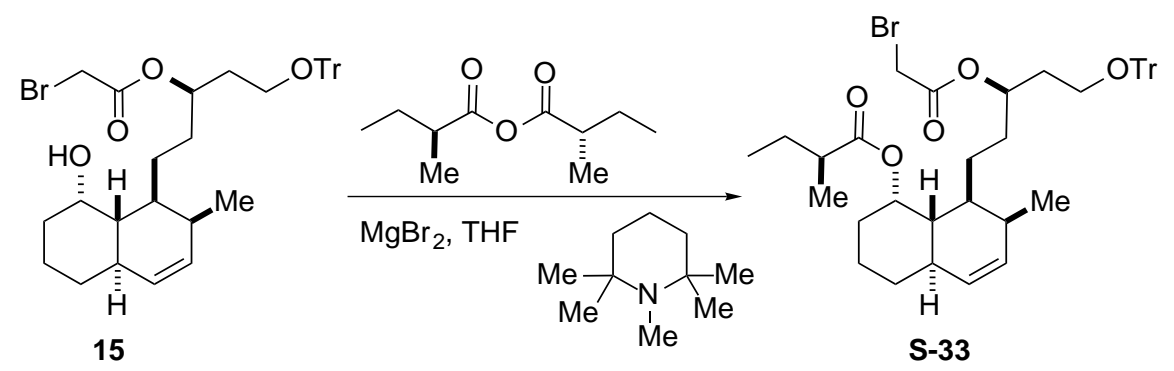

Ester S-33: 1,2-Dibromoethane (207 $\mu \mathrm{L}, 2.40 \mathrm{mmol})$ was added to magnesium turnings (50 mg, $2.06 \mathrm{mmol})$ in ether $(4 \mathrm{~mL})$, and the reaction was initiated by gentle heating until it became exothermic, at which point the heating mantle was removed. After 15 minutes, the heatng mantle was replaced and the reaction was heated to reflux for one hour after which time all of the magnesium turnings had been consumed.

In a separate flask, 1,2,2,6,6-pentamethylpiperidine (PMP) $(311 \mu \mathrm{L}, 1.72 \mathrm{mmol})$ was added to a solution of compound $15(0.271 \mathrm{~g}, 0.429 \mathrm{mmol})$ in THF $(1.4 \mathrm{~mL})$. Magnesium bromide (2.6 $\mathrm{mL}$, of the $0.5 \mathrm{M}$ solution prepared above, $1.29 \mathrm{mmol})$, was then added, followed by $(S)-(+)-2-$ methylbutyric anhydride (343 $\mu \mathrm{L}, 1.72 \mathrm{mmol}$, washed with saturated $\mathrm{NaHCO}_{3}$ and brine, dried over $\mathrm{MgSO}_{4}$, filtered through Celite, and concentrated under reduced pressure) and the reaction was stirred at room temperature for 1.5 hours. The reaction was then quenched by the addition of saturated $\mathrm{NaHCO}_{3}$, diluted with hexanes, and the layers separated. The aqueous layer was extracted with hexanes $(2 \times 5 \mathrm{~mL})$, and the combined organic layers were washed with brine, dried over $\mathrm{MgSO}_{4}$, filtered through Celite, and concentrated under reduced pressure. Purification by flash chromatography $\left(\mathrm{SiO}_{2}, 40: 1\right.$ hexanes/ethyl acetate) provided ester $\mathbf{S}-33$ (0.296 g, 0.414 mmol, $96 \%)$. TLC $\mathrm{R}_{\mathrm{f}}=0.34\left(10: 1\right.$ hexanes/ethyl acetate). ${ }^{1} \mathrm{H}$ NMR $\left(400 \mathrm{MHz}, \mathrm{CDCl}_{3}\right) \delta 7.41-7.38$ $(\mathrm{m}, 6 \mathrm{H}), 7.30-7.26(\mathrm{~m}, 6 \mathrm{H}), 7.23-7.19(\mathrm{~m}, 3 \mathrm{H}), 5.56(\mathrm{ddd}, J=2.6,4.6,9.8 \mathrm{~Hz}, 1 \mathrm{H}), 5.38(\mathrm{~d}, J=9.8$ $\mathrm{Hz}, 1 \mathrm{H}), 5.15(\mathrm{~s}, 1 \mathrm{H}), 5.05$ (pentet, $J=5.7 \mathrm{~Hz}, 1 \mathrm{H}), 3.69\left(\mathrm{H}_{\mathrm{a}}\right.$ of $\left.\mathrm{AB}, J=12.9,1 \mathrm{H}\right), 3.63\left(\mathrm{H}_{\mathrm{b}}\right.$ of $\mathrm{AB}$, $J=12.9,1 \mathrm{H}), 3.13-3.04(\mathrm{~m}, 1 \mathrm{H}), 2.32$ (sextet, $J=6.8 \mathrm{~Hz}, 1 \mathrm{H}), 2.28-2.17(\mathrm{~m}, 2 \mathrm{H}), 1.98$ (d, $J=14.4 \mathrm{~Hz}$, $1 \mathrm{H}), 1.86(\mathrm{q}, J=6.1 \mathrm{~Hz}, 2 \mathrm{H}), 1.73(\mathrm{~d}, J=12.9 \mathrm{~Hz}, 1 \mathrm{H}), 1.68-1.48(\mathrm{~m}, 6 \mathrm{H}), 1.46-1.31(\mathrm{~m}, 4 \mathrm{H}), 1.16$ (td, $J=10.5,1.3 \mathrm{~Hz}, 1 \mathrm{H}), 1.09(\mathrm{~d}, J=6.8 \mathrm{~Hz}, 3 \mathrm{H}), 1.04(\mathrm{td}, \mathrm{J}=11.5,4.0 \mathrm{~Hz}, 2 \mathrm{H}), 0.86(\mathrm{t}, J=7.6,3 \mathrm{H})$, $0.77(\mathrm{~d}, J=6.9 \mathrm{~Hz}, 3 \mathrm{H}) .{ }^{13} \mathrm{C} \mathrm{NMR}\left(100 \mathrm{MHz} \mathrm{CDCl}_{3}\right) \delta 176.07,166.74,144.21,132.34,131.09$, 128.86, 127.97, 127.13, 86.77, 74.50, 68.96, 59.74, 42.12, 41.96, 37.71, 36.90, 33.88, 33.02, 31.53, $31.21,30.88,27.05,26.64,22.37,21.13,17.08,15.06,11.93$. IR 2936, 2876, 1729, 1450, 1375, $1276,1166,1077,909,714,416 \mathrm{~cm}^{-1} .[\alpha]_{\mathrm{D}^{20}}=+43.89(c=2.11)$. Exact mass calculated for $\left[\mathrm{C}_{42} \mathrm{H}_{51} \mathrm{BrO}_{5} \mathrm{Na}\right]^{+}:$737.28120. Found: 737.28294 (ESI).

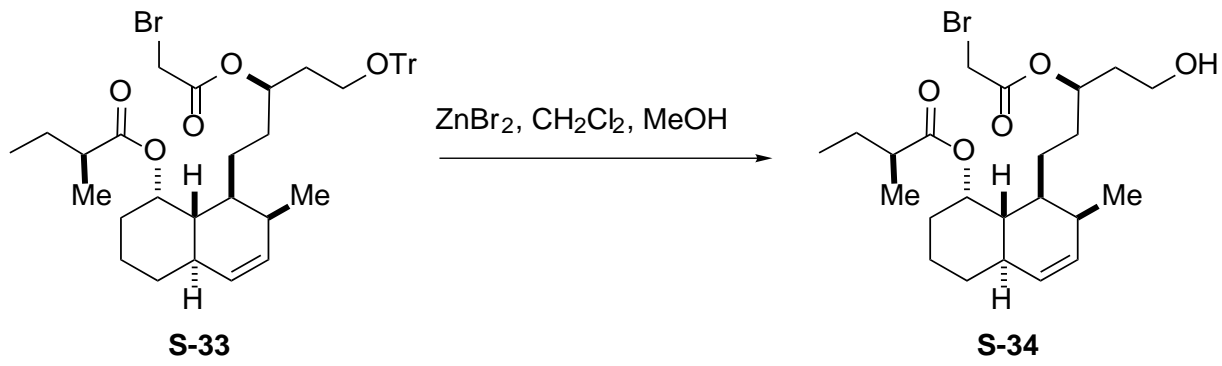

Alcohol S-34: A solution of compound S-33 (0.280 g, $0.391 \mathrm{mmol})$ in $\mathrm{CH}_{2} \mathrm{Cl}_{2}(3 \mathrm{~mL})$ was added via cannula to a rapidly stirring mixture of anhydrous zinc bromide $(3.52 \mathrm{~g}, 1.56 \mathrm{mmol})$ in methanol $(1 \mathrm{~mL})$ and $\mathrm{CH}_{2} \mathrm{Cl}_{2}(1 \mathrm{~mL})$. The reaction was stirred at room temperature for 4 hours, 
and quenched by the addition of saturated $\mathrm{NaHCO}_{3}(15 \mathrm{~mL})$. The organic layer was washed with $\mathrm{NaHCO}_{3}(3 \times 5 \mathrm{~mL})$ and water $(3 \times 5 \mathrm{~mL})$. The combined aqueous layers were extracted with ethyl acetate $(3 \times 5 \mathrm{~mL})$, and the combined organic layers were washed with brine $(5 \mathrm{~mL})$, dried over $\mathrm{MgSO}_{4}$, filtered through Celite, and concentrated under reduced pressure. Purified by flash chromatography $\left(\mathrm{SiO}_{2}, 5: 1\right.$ hexanes/ethyl acetate) provided alcohol $\mathbf{S - 3 4}(158 \mathrm{mg}, 0.334 \mathrm{mmol}$, $85 \%) . \quad$ TLC $\mathrm{R}_{\mathrm{f}}=0.53\left(2: 1\right.$ hexanes/ethyl acetate). ${ }^{1} \mathrm{H}$ NMR $\left(500 \mathrm{MHz}, \mathrm{CDCl}_{3}\right) \delta 5.57$ (ddd, $J=$ $2.84 .8,9.8 \mathrm{~Hz}, 1 \mathrm{H}), 5.39(\mathrm{~d}, J=9.8 \mathrm{~Hz}, 1 \mathrm{H}), 5.16-5.14(\mathrm{~m}, 1 \mathrm{H}), 5.02-4.97(\mathrm{~m}, 1 \mathrm{H}), 3.83(\mathrm{~s}, 2 \mathrm{H})$, 3.68-3.62 (m, 1H), 3.60-3.54 (m, 1H), 2.34 (sextet, $J=7.0 \mathrm{~Hz}, 1 \mathrm{H}), 2.27-2.22(\mathrm{~m}, 2 \mathrm{H}), 1.98$ (dd, $J=5.3,7.0 \mathrm{~Hz}, 1 \mathrm{H}), 1.95$ (br. s, $1 \mathrm{H}), 1.86-1.97(\mathrm{~m}, 1 \mathrm{H}), 1.78-1.62(\mathrm{~m}, 4 \mathrm{H}), 1.62-1.52(\mathrm{~m}, 2 \mathrm{H})$, 1.51-1.32 (m, 5H), $1.18(\mathrm{td}, J=10.7,1.6 \mathrm{~Hz}, 1 \mathrm{H}), 1.15-1.07(\mathrm{~m}, 1 \mathrm{H}), 1.12(\mathrm{~d}, \mathrm{~J}=7.0 \mathrm{~Hz}, 3 \mathrm{H}), 1.02$ (ddd, $J=12.4,4.4 \mathrm{~Hz}, 1 \mathrm{H}), 0.89$ (t, $J=7.4,3 \mathrm{H}), 0.81$ (d, $J=7.0 \mathrm{~Hz}, 3 \mathrm{H}) .{ }^{13} \mathrm{C}$ NMR $(100 \mathrm{MHz}$, $\left.\mathrm{CDCl}_{3}\right) \delta 176.24,167.93,132.26,131.14,74.58,69.01,58.85,42.15,41.97,37.68,37.10,36.89$, $33.01,31.23,31.60,30.93,27.06,26.22$, 23.12, 21.13, 17.10, 15.05, 11.94. IR 3443, 2936, 2877, $1725,1451,1279,1178,1066,969 \mathrm{~cm}^{-1}$. [ $\left.\alpha\right]_{\mathrm{D}^{20}=+72.5}(c=1.20)$. Exact mass calculated for $\left[\mathrm{C}_{23} \mathrm{H}_{37} \mathrm{BrO}_{5} \mathrm{Na}\right]^{+}:$495.17165. Found: 495.17128 (ESI).
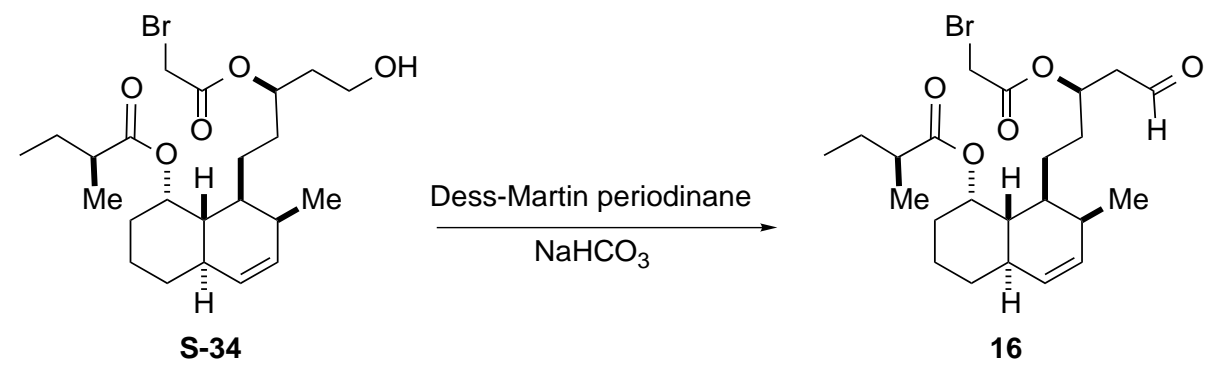

Aldehyde 16: Solid $\mathrm{NaHCO}_{3}(0.360 \mathrm{~g}, 4.29 \mathrm{mmol})$ was added to a solution of alcohol S$34(0.154 \mathrm{~g}, 0.326 \mathrm{mmol})$ in $\mathrm{CH}_{2} \mathrm{Cl}_{2}(3 \mathrm{~mL})$. The Dess-Martin Periodinane $(0.180 \mathrm{~g}, 0.424 \mathrm{mmol})$ was then added and the reaction was stirred for 1 hour at room temperature, then quenched with an aqueous $\mathrm{NaHCO}_{3} / \mathrm{Na}_{2} \mathrm{~S}_{2} \mathrm{O}_{3}$ solution (5 mL, 2:1:1 saturated $\mathrm{NaHCO}_{3}$, saturated $\mathrm{Na}_{2} \mathrm{~S}_{2} \mathrm{O}_{3}$, water). The organic layer was washed with the $\mathrm{NaHCO}_{3} / \mathrm{Na}_{2} \mathrm{~S}_{2} \mathrm{O}_{3}$ solution $(5 \mathrm{~mL})$ and the combined aqueous layers were extracted with ether $(3 \times 5 \mathrm{~mL})$. The combined organic layers were washed with water $(5 \mathrm{~mL})$ and brine $(5 \mathrm{~mL})$, dried over $\mathrm{MgSO}_{4}$, filtered through Celite, and concentrated under reduced pressure. The product was filtered through neutral silica gel (5:1 hexanes/ether) to provide aldehyde $16(135 \mathrm{mg}, 0.286 \mathrm{mmol}, 88 \%)$ as a white foam which was used immediately in the next reaction. TLC $\mathrm{R}_{\mathrm{f}}=0.44$ (3:1 hexanes/ethyl acetate). ${ }^{1} \mathrm{H}$ NMR $\left(500 \mathrm{MHz}, \mathrm{CDCl}_{3}\right) \delta 9.71$ $(\mathrm{m}, 1 \mathrm{H}), 5.56$ (ddd, $J=2.74 .7,9.6 \mathrm{~Hz}, 1 \mathrm{H}), 5.39(\mathrm{~d}, J=10.1 \mathrm{~Hz}, 1 \mathrm{H}), 5.26$ (pentet, $J=6.0 \mathrm{~Hz}, 1 \mathrm{H}$ ), 5.17-5.15 (m, 1H), $3.80(\mathrm{~s}, 2 \mathrm{H}), 2.72(\mathrm{ABX}, J=2.3,7.4,17.1 \mathrm{~Hz}, 1 \mathrm{H}), 2.66(\mathrm{ABX}, \mathrm{J}=1.0,5.1,17.1$ $\mathrm{Hz}, 1 \mathrm{H}), 2.34$ (sextet, $J=7.1 \mathrm{~Hz}, 1 \mathrm{H}), 2.27-2.23(\mathrm{~m}, 2 \mathrm{H}), 1.96(\mathrm{~d}, J=15 \mathrm{~Hz}, 1 \mathrm{H}), 1.80-1.36(\mathrm{~m}$, $10 \mathrm{H}), 1.30-1.06(\mathrm{~m}, 2 \mathrm{H}), 1.12(\mathrm{~d}, J=7.1 \mathrm{~Hz}, 3 \mathrm{H}), 1.02(\mathrm{qd}, \mathrm{J}=12.2,4.5,1 \mathrm{H}), 0.89$ (t, $J=7.4,3 \mathrm{H})$, $0.80(\mathrm{~d}, J=7.1 \mathrm{~Hz}, 3 \mathrm{H})$.
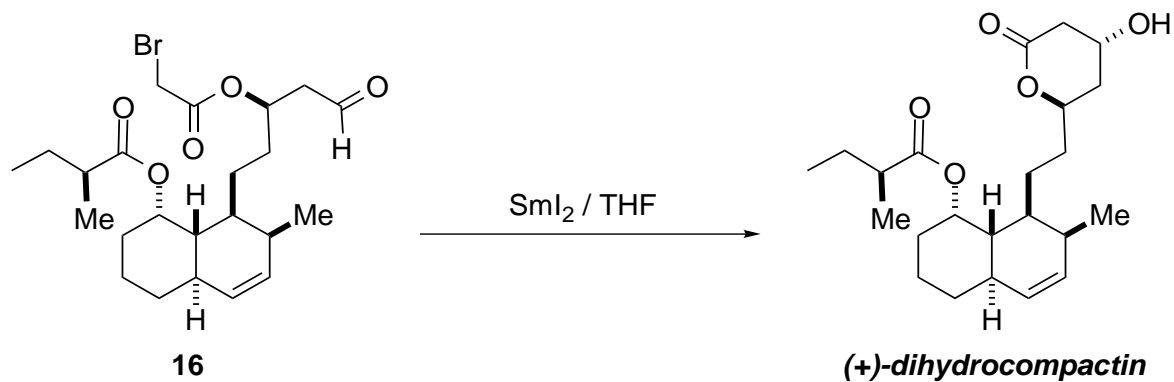

(+)-Dihydrocompactin: A solution of 1,2-diiodoethane $(0.373 \mathrm{~g}, 1.32 \mathrm{mmol}$, purified by washing an ether solution with saturated $\mathrm{Na}_{2} \mathrm{~S}_{2} \mathrm{O}_{3}$, and brine, drying over $\mathrm{MgSO}_{4}$, filtering and 
concentrating under reduced pressure. This material was further dried under high vacuum, and protected from light by wrapping the flask in aluminum foil) in THF (2.6 mL) was added to a mixture of samarium powder $(0.181 \mathrm{~g}, 1.20 \mathrm{mmol})$ in THF $(1 \mathrm{~mL})$. The reaction mixture was protected from light, and stirred for 4 hours at room temperature at which point it was a deep blue color and was cooled to $-100{ }^{\circ} \mathrm{C}$. Aldehyde $16(100.5 \mathrm{mg}, 0.213 \mathrm{mmol})$ in THF $(1 \mathrm{~mL})$ was added to this solution dropwise via cannula, and the reaction was maintained between $-80{ }^{\circ} \mathrm{C}$ and $-100{ }^{\circ} \mathrm{C}$ for 14 hours. It was then quenched by the addition of saturated $\mathrm{NH}_{4} \mathrm{Cl}(3 \mathrm{~mL})$, warmed to room temperature, and diluted with ethyl acetate. The organic layer was washed with $\mathrm{NH}_{4} \mathrm{Cl}(4 \times 5 \mathrm{~mL})$, water $(3 \times 5 \mathrm{~mL})$, brine $(5 \mathrm{~mL})$, dried over $\mathrm{MgSO}_{4}$, filtered through Celite, and concentrated under reduced pressure. The product was purified by flash chromatography $\left(\mathrm{SiO}_{2}, 5: 1\right.$ to $2: 1$ hexanes/ethyl acetate) to provide (+)-dihydrocompactin $(58.2 \mathrm{mg}, 0.148 \mathrm{mmol}, 70 \%)$ as a white solid with a melting point of $84-87{ }^{\circ} \mathrm{C}$. Recrystallization from hexanes $/ \mathrm{CH}_{2} \mathrm{Cl}_{2}$ /ether provided white needles melting at $144-145^{\circ} \mathrm{C}$. TLC $\mathrm{R}_{\mathrm{f}}=0.52$ (ethyl acetate). ${ }^{1} \mathrm{H}$ NMR $\left(500 \mathrm{MHz}, \mathrm{CDCl}_{3}\right)$ $\delta 5.58(\mathrm{ddd}, J=2.4,4.6,9.7 \mathrm{~Hz}, 1 \mathrm{H}), 5.39$ (d, $J=9.7 \mathrm{~Hz}, 1 \mathrm{H}), 5.16$ (br. s, $1 \mathrm{H}), 4.62-4.57$ (m, $1 \mathrm{H})$, 4.34 (br s, 1H), 2.70 (A of ABX, $J=1,4.9,17.5 \mathrm{~Hz}, 1 \mathrm{H}), 2.60$ (B of ABX, $J=1.5,3.6,17.5 \mathrm{~Hz}, 1 \mathrm{H}$ ), 2.35 (sextet, $J=7.0 \mathrm{~Hz}, 1 \mathrm{H}), 2.28-2.23(\mathrm{~m}, 3 \mathrm{H}), 2.10(\mathrm{~m}, 1 \mathrm{H}), 1.99-1.92(\mathrm{~m}, 2 \mathrm{H}), 1.88-1.80(\mathrm{~m}$, 1H), 1.73 (br d, J=12.8 Hz, 1H), 1.68-1.59 (m, 3H), $1.58-1.35(\mathrm{~m}, 5 \mathrm{H}), 1.31-1.25(\mathrm{~m}, 2 \mathrm{H}), 1.20$ $(\mathrm{t}, J=10.6,2 \mathrm{H}), 1.12(\mathrm{~d}, J=7.0 \mathrm{~Hz}, 3 \mathrm{H}), 1.03(\mathrm{qd}, J=4.6,12.7 \mathrm{~Hz}, 1 \mathrm{H}), 0.89(\mathrm{t}, J=7.6,3 \mathrm{H}), 0.82(\mathrm{~d}$, $J=7.0 \mathrm{~Hz}, 3 \mathrm{H}) .{ }^{13} \mathrm{C}$ NMR $\left(100 \mathrm{MHz}, \mathrm{CDCl}_{3}\right) \delta 176.49,170.52,132.37,131.11,76.41,69.18$, 62.90, 42.16, 41.93, 38.78, 37.89, 36.99, 36.28, 33.22, 32.96, 31.69, 30.90, 27.02, 23.42, 21.13, 17.16, 15.04, 11.97. IR 3447, 2935, 2877, 1721, 1449, 1374, 1251, 1194, 1067, $731 \mathrm{~cm}^{-1}$. $[\alpha]_{\mathrm{D}}^{20}=$ +123.3 (c=1.08). Exact mass calculated for $\left[\mathrm{C}_{23} \mathrm{H}_{36} \mathrm{O}_{5} \mathrm{Na}\right]^{+}: 415.24549$. Found: 415.24562 (ESI). This material displayed identical spectral properties to that described in the literature and was further characterized by gDQCOSY, gHSQC, gHMBC, NOESY, and gradient NOE spectroscopy.

Comments on the molecular modeling: A Monte-Carlo conformational search was performed using the semi-empirical AM1 method as implemented in Spartan Pro (Wavefunction Inc., Irvine, CA.). Five conformers were found within $1.4 \mathrm{kcal} / \mathrm{mol}$ of the global minimum, other conformers were at least $4.4 \mathrm{kcal} / \mathrm{mol}$ higher than the global minimum and were ignored. The ethyl sidechain in the three lowest energy conformers (relative $\mathrm{E}=0,0.32$, and $0.92 \mathrm{kcal} / \mathrm{mol}$ ) exists in the pseudo-equatorial conformation. They differ by rotation about the $\mathrm{C}-\mathrm{C}$ bond of the ethyl group. In the remaining two conformers (relative $\mathrm{E}=1.12$, and $1.33 \mathrm{kcal} / \mathrm{mol}$ ) the ethyl side chain is pseudo-axial, and they also differ by rotation of the $\mathrm{C}-\mathrm{C}$ bond of the ethyl group. An overlay of these conformers (Figure 1) shows remarkably little difference in the conformation of the ring in each set of conformations.

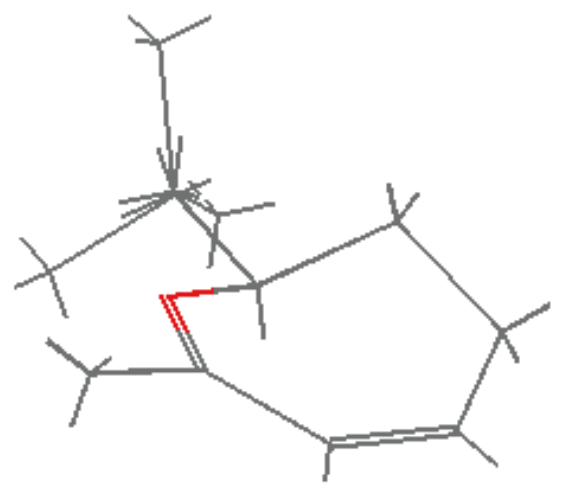

Overlay of the three lowest energy conformers Figure 1 (ethyl group is pseudo-equatorial)

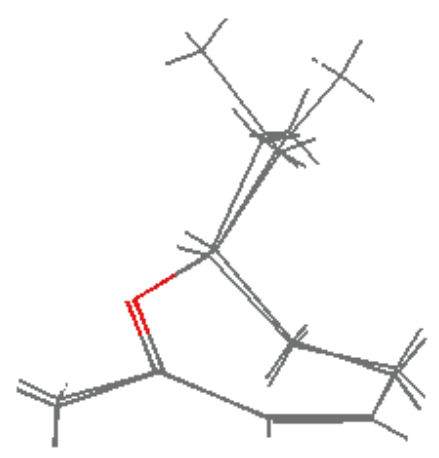

Overlay of the remaining two conformers (ethyl group is pseudo-axial) 